\title{
Metallicity distributions of mono-age stellar populations of the Galactic disc from the LAMOST Galactic spectroscopic surveys
}

\author{
C. Wang ${ }^{1 \star}$, X.-W. Liu ${ }^{1,2,3 \star}$, M.-S. Xiang ${ }^{4,5} \dagger$, Y. Huang ${ }^{1,3} \dagger$, B.-Q. Chen ${ }^{3} \dagger$, H.-B. Yuan ${ }^{6}$, \\ J.-J. Ren ${ }^{4}$, H.-W. Zhang ${ }^{1,2}$, Z.-J. Tian ${ }^{1} \dagger$ \\ ${ }^{1}$ Department of Astronomy, Peking University, Beijing 100871, People's Republic of China. \\ ${ }^{2}$ Kavli Institute for Astronomy and Astrophysics, Peking University, Beijing 100871, People's Republic of China. \\ ${ }^{3}$ South-Western Institute for Astronomy Research, Yunnan University, Kunming, Yunnan 650091, People's Republic of China. \\ ${ }^{4}$ National Astronomical Observatories, Chinese Academy of Sciences, Beijing 100012, People's Republic of China. \\ ${ }^{5}$ Max-Planck Institute for Astronomy, Konigstuhl, D-69117, Heidelberg, Germany. \\ ${ }^{6}$ Department of Astronomy, Beijing Normal University, Beijing 100875, People's Republic of China.
}

20180530

\begin{abstract}
We have investigated the metallicity distributions of mono-age stellar populations across the disc of $6 \lesssim R \lesssim 12 \mathrm{kpc}$ and $|Z| \lesssim 2 \mathrm{kpc}$ using samples selected from the main-sequence turnoff and sub-giant (MSTO-SG) stars targeted by the LAMOST Galactic Spectroscopic surveys. Both the mean values and the profiles of the distributions exhibit significant variations with age and position. We confirm that the oldest $(>11 \mathrm{Gyr})$ stars have nearly flat radial $[\mathrm{Fe} / \mathrm{H}]$ gradients at all heights above the disc but show negative vertical $[\mathrm{Fe} / \mathrm{H}]$ gradients. For stars younger than $11 \mathrm{Gyr}$, the radial $[\mathrm{Fe} / \mathrm{H}]$ gradients flatten with $|Z|$, while the vertical $[\mathrm{Fe} / \mathrm{H}]$ gradients flatten with $R$. Stars of 4-6 Gyr exhibit steeper negative radial $[\mathrm{Fe} / \mathrm{H}]$ gradients than those of either younger or older ages. Values of $[\alpha / \mathrm{Fe}]$ of mono-age stellar populations also show significant radial and vertical gradients, with patterns varying with age. The $[\mathrm{Fe} / \mathrm{H}]$ distribution profiles of old ( $>8 \mathrm{Gyr}$ ) stars vary little with $R$, while those of younger stars exhibit strong radial variations, probably a consequence of significant radial migration. The $[\alpha / \mathrm{Fe}]$ radial distribution profiles show opposite patterns of variations with age compared to those of $[\mathrm{Fe} / \mathrm{H}]$. We have also explored the impacts of stellar mixing by epicycle motions (blurring) on the $[\mathrm{Fe} / \mathrm{H}]$ and $[\alpha / \mathrm{Fe}]$ distributions, and found that blurring mainly change the widths of the distribution profiles. Our results suggest that the disc may have experienced a complex assemblage history, in which both the "inside-out" and "upside-down" formation processes may have played an important role.
\end{abstract}

Key words: Galaxy: abundances - Galaxy: disc - Galaxy: structure - Galaxy: forming Galaxy: evolution

\section{INTRODUCTION}

As a typical disc galaxy, our own galaxy the Milky Way (MW) is an excellent laboratory to test the galaxy formation and evolution scenarios with the advantage that the individual stars in the MW can be resolved. Since most of the MW's baryons and angular momentum are in the disc, the knowledge of how the disc was formed and assembled is a key to understanding the formation and evolution of our Galaxy. However, many questions regarding the disc formation and assemblage remain open, and there is great debate about this topic (e.g. Rix \& Bovy 2013).

The surface chemical composition of low- and intermediatemass stars remains almost unchanged during the main-sequence

^ E-mails: wchun@pku.edu.cn (CW); x.liu@pku.edu.cn (XWL)

$\dagger$ LAMOST Fellow and turn-off evolutionary stage, which makes it fossil record of the environment of the interstellar medium at the position and time of the births of those stars. The stellar number density $N$ as a function of metallicity $[\mathrm{Fe} / \mathrm{H}]$ and $\alpha$-element to iron abundance ratio $[\alpha / \mathrm{Fe}]$ at a given disc position of radius $R$ and height $Z$ and age $\tau$, the socalled stellar metallicity distribution function, $\mathrm{MDF} \equiv N([\mathrm{Fe} / \mathrm{H}]$, $[\alpha / \mathrm{Fe}] \mid R, Z, \tau)$, is the consequence of many complex physical processes, including the infall and outflow of gas (Larson 1976 , Colavitti, Matteucci \& Murante 2008, Pezzulli \& Fraternali 2016, Andrews et al. 2017, Toyouchi \& Chiba 2018, Grisoni, Spitoni \& Matteucci 2018), the supernova feedback (Kobayashi \& Nakasato 2011), the secular evolution (e.g. radial migration; Sellwood \& Binney 2002, Roškar et al. 2008. Schönrich \& Binney 2009, Loebman et al. 2011, Haywood et al. 2016), the non-axisymmetric perturbations (e.g. by the central bar or spiral arms), and the accretions of dwarf galaxies (Quinn, Hernquist \& Fullagar 1993). The stellar 
Wang et al.

metallicity distributions therefore provide strong constraints on the Galactic disc formation and evolution history, especially on when, where and how much those processes affect the assemblage and evolution of the Galactic disc.

The gradients of $[\mathrm{Fe} / \mathrm{H}]$ and $[\alpha / \mathrm{Fe}]$ represent the first-order variations with spatial position (the slopes) of the zero-order MDF (the mean values). The $[\mathrm{Fe} / \mathrm{H}]$ gradients in the radial and vertical directions have been determined with a variety of tracers, including OB stars (Daflon \& Cunha 2004), Cepheid variables (Andrievsky et al. 2002, Luck, Kovtyukh \& Andrievsky 2006), H II regions (Balser et al. 2011), open clusters (Chen, Hou \& Wang 2003. Magrini et al. 2009), planetary nebulae (Costa, Uchida \& Maciel 2004. Henry et al. 2010) , FGK dwarfs (Katz et al. 2011; Cheng et al. 2012, Boeche et al. 2013), (red) giant stars (Hayden et al. 2014. Boeche et al. 2014) and red clump stars (Huang et al. 2015). The results reveal that the disc has negative gradients in both radial and vertical directions. The gradients have also been demonstrated to exhibit significant spatial variations. The observed negative radial $[\mathrm{Fe} / \mathrm{H}]$ gradients generally support the "inside-out" disc forming scenario. On the other hand, none of the above studies clearly show how the $[\mathrm{Fe} / \mathrm{H}]$ gradients vary among different stellar populations due to the lack of robust age estimates for a large sample of stars. In a pioneering study, Xiang et al. (2015b) determine the radial and vertical $[\mathrm{Fe} / \mathrm{H}]$ gradients of mono-age stellar populations using a sample of 0.3 million main-sequence turn-off (MSTO) stars with robust age estimates selected from the LAMOST Spectroscopic Surveys of the Galactic Anti-centre (LSS-GAC; Liu et al. 2014, Yuan et al. 2015). They find that both the radial and vertical gradients show strong variations among different mono-age stellar populations. The radial gradients are very small for the oldest populations, and become steeper as age decreases, reaching a maximum (steepest) at age $6-8 \mathrm{Gyr}$, and then flatten again with decreasing age. The vertical gradients generally flatten with decreasing age for $\tau<11$ Gyr. Their results suggest that the disc may have experienced at least two different assemblage phases.

The MDF profiles contain rich information of the Galactic disc assemblage history. The profile of the $[\mathrm{Fe} / \mathrm{H}]$ distribution in the solar neighbourhood has been widely studied (e.g. van den Bergh 1962, Nordström et al. 2004, Casagrande et al. 2011). The results show very broad $[\mathrm{Fe} / \mathrm{H}]$ profiles at the solar neighbourhood, suggesting that both gas dynamics and stellar migration have played important roles in the disc assemblage history. Beyond the solar neighbourhood, the variations of the $[\mathrm{Fe} / \mathrm{H}]$ distribution with positions have been investigated with data from the RAVE (Siebert et al. 2011), SEGUE (Schlesinger et al. 2012) and APOGEE (Anders et al. 2014. Hayden et al.2015) surveys. The results show that the profile of the $[\mathrm{Fe} / \mathrm{H}]$ distribution varies with both $R$ and $Z$. Hayden et al. (2015) demonstrate that the low- $[\alpha / \mathrm{Fe}]$ populations close to the Galactic plane have negatively skewed $[\mathrm{Fe} / \mathrm{H}]$ distributions in the inner disc and positively skewed distributions in the outer disc, a result that they interpret as a consequence of stellar radial migration, in particular that induced by the churning process. Their results also show that high- $[\alpha / \mathrm{Fe}]$ populations exhibit a similar profile at all positions. The different behaviours of the MDF between the low- $[\alpha / \mathrm{Fe}]$ and high- $[\alpha / \mathrm{Fe}]$ populations suggest that the profile of $[\mathrm{Fe} / \mathrm{H}]$ distribution must vary with population since $[\alpha / \mathrm{Fe}]$ is a rough indicator of stellar age. $[\alpha / \mathrm{Fe}]$ however can only be used to differentiate stars into two populations: a young and an old population, insufficient to study in detail how the strength of radial migration varies with time throughout the whole assemblage history of the disc. A large sample of stars with robust age estimates is essential to clarify this important issue.
Recently, robust ages for a million main-sequence turn-off and sub-giant stars (MSTO-SG), selected from the LAMOST Galactic spectroscopic surveys (Cui et al. 2012, Zhao et al. 2012, Deng et al. 2012, Liu et al. 2014, Yuan et al. 2015), have become available (Xiang et al. 2017a). The sample stars span a wide range of age, cover a large and contiguous volume of the Galactic disc and have a simple yet non-trivial uniform target selection function. The sample provides an excellent opportunity to characterise the MDF of mono-age stellar populations, and understand the disc assemblage history in more detail. In this work, we present the MDF of mono-age stellar populations across the disc of $6 \lesssim R \lesssim 12 \mathrm{kpc}$ and $|Z| \lesssim 2 \mathrm{kpc}$. We have derived the distribution function not only of $[\mathrm{Fe} / \mathrm{H}]$, but also of $[\alpha / \mathrm{Fe}]$. Although there have been some work focusing on the distributions of $[\alpha / \mathrm{H}]$ (Genovali et al. 2015, Hayden et al. 2015) and [ $\alpha / \mathrm{Fe}$ (Anders et al. 2014, Boeche et al. 2014), the study is not as comprehensive as that of $[\mathrm{Fe} / \mathrm{H}]$. In the current work, we investigate the spatial variations of both the gradients and profiles of the $[\mathrm{Fe} / \mathrm{H}]$ and $[\alpha / \mathrm{Fe}]$ distributions for different monoage stellar populations, and explore the impacts of stellar mixing by epicycle motions (blurring) on the distributions. Amongst them, the radial and vertical $[\alpha / \mathrm{Fe}]$ gradients and the profiles of $[\mathrm{Fe} / \mathrm{H}]$ and $[\alpha / \mathrm{Fe}]$ distributions of mono-age stellar populations are investigated for the first time.

This paper is organised as follows. In Section 2, we briefly introduce the MSTO-SG sample and corrections for the selection effects. In Section 3, we present the $[\mathrm{Fe} / \mathrm{H}]$ and $[\alpha / \mathrm{Fe}]$ gradients of mono-age stellar populations. We present the metallicity distribution profiles of mono-age stellar populations in Section 4. The effects of the blurring process on the metallicity distributions are discussed in Section 5. The constraints of the MDF derived here on the formation and evolution of the Galactic disc are discussed in Section 6. Finally, we summary our work in Section 7.

\section{DATA}

\subsection{The LAMOST MSTO-SG star sample}

The sample used in the current work consists of 0.93 million MSTO-SG stars with robust age estimates selected from the Pilot Surveys and the first four years of five-year Phase I Regular Surveys of the LAMOST Galactic spectroscopic surveys (Xiang et al.2017a). Several studies have been devoted to derive robust estimates of stellar atmospheric parameters from the LAMOST spectra, such as the LAMOST Stellar Parameter Pipeline (LASP; Luo et al. 2015), the LAMOST Stellar Parameter Pipeline at Peking University (LSP3; Xiang et al. 2015a. 2017b), applying the SEGUE Stellar Parameter Pipeline (SSPP) to LAMOST spectra (Lee et al. 2015), parameter determinations based on the Cannon (Ho et al. 2017) and an analysis based on the SP_Ace code (Boeche et al. 2018). The sample of MSTO-SG stars of Xiang et al. (2017a) was constructed based on LSP3 stellar parameters (effective temperature $T_{\text {eff }}$, surface gravity $\log g$, metallicity $[\mathrm{Fe} / \mathrm{H}], \alpha$-element abundance ratio $[\alpha / \mathrm{Fe}]$, radial velocity $V_{r}$, absolute magnitudes $M_{\mathrm{V}}$ and $M_{\mathrm{Ks}}$ ). Typical uncertainties of the parameters are listed in Table 1 for SNRs higher than 20 and 50. Note that all the MSTOSG sample stars of Xiang et al. (2017a) have SNRs higher than 20. Stellar distances are inferred from the distance modulus using the absolute magnitudes derived directly from the spectra, and utilising interstellar extinction derived with the 'star pair' method (Yuan, Liu \& Xiang 2013, Yuan et al. 2015, Xiang et al. 2017b). The average distance uncertainty of the sample stars is 17 percent. Ages of the 

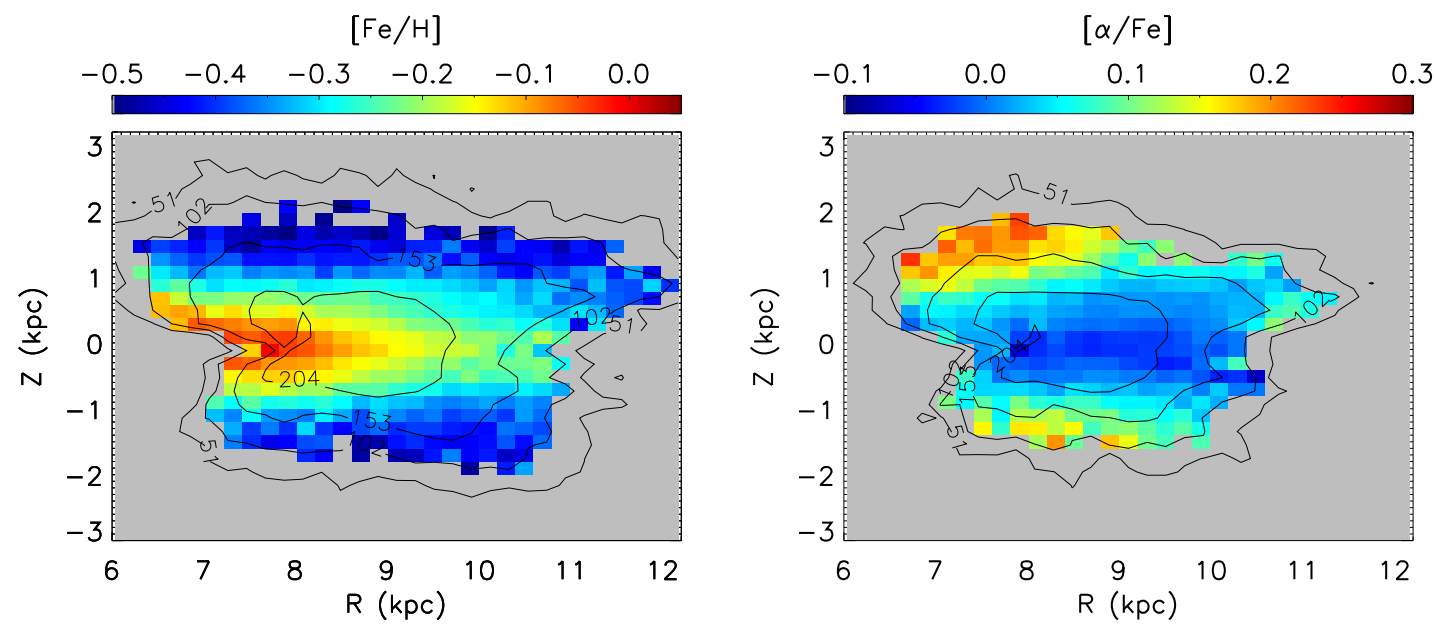

Figure 1. $[\mathrm{Fe} / \mathrm{H}]$ (left panel) and $[\alpha / \mathrm{Fe}]$ (right panel) distributions of the MSTO-SG-SNR20 (left panel) and MSTO-SG-SNR50 (right panel) samples, binned by $0.2 \times 0.2 \mathrm{kpc}$ in the $R-Z$ plane. The over-plotted contours show the corresponding density (star number count in each $R-Z$ bin) distributions on logarithmic scale.

stars are determined by matching with stellar isochrones using a Bayesian algorithm, as detailed in Xiang et al. (2017a). The derived ages are shown to be accurate to better than 30 per cent via a series of internal and external comparisons.

In this work, we discard MSTO-SG stars of $T_{\text {eff }}>7500 \mathrm{~K}$ and $M_{\mathrm{V}}>4.5 \mathrm{mag}$ as they have relatively large uncertainties in their $[\mathrm{Fe} / \mathrm{H}]$ estimates, which is used to investigate the $[\mathrm{Fe} / \mathrm{H}]$ distributions. Here we refer to this sample as "MSTO-SG-SNR20". Moreover, the study of $[\alpha / \mathrm{Fe}]$ distributions of mono-age stellar populations requires higher SNRs because the $[\alpha / \mathrm{Fe}]$ measurements are more sensitive to spectral SNRs. For this purpose, we further define a sub-sample with spectral SNRs $>50$. We refer this sample to "MSTO-SG-SNR50". However, the $[\alpha / \mathrm{Fe}]$ estimates from LSP3 for relatively hot (e.g. > $6000 \mathrm{~K}$ ) thus young stars may suffer large uncertainties due to inadequacies of the adopted template spectra as well as the intrinsic weak spectral features of $\alpha$ elements. In addition, Xiang et al. (2017a) suggest that about 10-20 per cent of stars younger than $4 \mathrm{Gyr}$ may be blue stragglers whose ages have been wrongly estimated. Therefore, one needs to be cautious about the derived $[\alpha / \mathrm{Fe}]$ gradients and distribution profiles of the young populations in the current work.

Compared to the MSTO star sample used by Xiang et al. (2015b) for the study of the Galactic disc's metallicity gradients, the current MSTO-SG star sample has been significantly improved in terms of that, besides the significantly increase of sample size and the inclusion of $[\alpha / \mathrm{Fe}]$ which is not available in Xiang et al. (2015b), systematic errors in age estimates are reduced due to improvements in stellar parameter determinations. As a consequence, potential sources of contamination are also better understood and well-controlled. Xiang et al. (2015b) selected MSTO in the $T_{\text {eff }}$ $\log g$ plane (see Fig. 1 of Xiang et al. (2015b) ), using parameters derived with the LSP3 version for the LSS-GAC DR1. While the $\log g$ estimates then suffer from significant (systematic) uncertainties (Ren et al. 2016). Xiang et al. (2017a) have selected the MSTO-SG stars in the $T_{\text {eff }}-\mathrm{M}_{V}$ plane (see their Fig. 5). Values of $\mathrm{M}_{V}$ have been derived directly from the LAMOST spectra with a machine learning method based on kernel-based component analysis utilising the LAMOST-Hipparcos common stars as a training dataset, and the systematic errors are found to be negligible (Xiang et al. $2017 \mathrm{a} \mid \mathrm{b}$ ). Nevertheless, we note that despite all these im- provements, the current MSTO-SG sample stars may still suffer from contaminations from dwarf, giant, supergiant and binary stars due to random errors in the stellar parameters. The contaminations could be severe and non-negligible for the old stellar populations.

\subsection{Selection effect correction}

Nandakumar et al. (2017) and Chen et al. (2018) have suggested that the LAMOST selection function has only a marginal effect on the the peak of the metallicity distribution. Nevertheless, both Nandakumar et al. (2017) (see their Fig. 10) and Chen et al. (2018) (see their Fig. 11) do find that the dispersion and skewness of the MDF are affected by the selection effects of LAMOST. The possible effects of the selection effects of LAMOST on the MDF of mono-age stellar populations remain to be investigated. To clarify the situation, we have considered and corrected for the selection function of our sample stars.

Generally, the selection effects of our sample concerned in the current work mainly consist of two parts: (1) the selection effects of the LAMOST Galactic spectroscopic surveys with respect to the photometric catalogues from which the spectroscopic targets are selected. The latter is usually assumed to be complete (to a certain limiting magnitude) and represents the underlying stellar populations; and (2) the incompleteness of MSTO-SG stars in a given volume caused by the limiting magnitude of the surveys and the wide range of absolute magnitudes of MSTO-SG stars. Such an effect also varies with stellar populations of different ages, as they have different intrinsic width of absolute magnitude range according to their definition (Xiang et al.2017a). The first part is corrected using the selection function $S$ derived by Chen et al. (2018). Based on the source of origin, $S$ contains also two portions. The first portion, quantified by $S_{1}$, characterises the LAMOST target selection strategy. The second portion, quantified by $S_{2}$, characterises the selection effects due to the observational quality, data reduction and parameter determination. Each star in our sample is weighted by $\frac{1}{S}$ in the colour-magnitude diagram (CMD). In order to account for the volume incompleteness of the mono-age populations of MSTO-SG stars, we define the completeness volumes of our sample stars using the same method of Xiang et al. (2018) and Chen et al. (2017). The complete volume of a sample of stars is a combined consequence 
Table 1. Typical accuracies of atmospheric parameters determined with LSP3 for stars of spectral SNRs $>20$ and $>50$.

\begin{tabular}{cccccccc}
\hline & $T_{\text {eff }}(\mathrm{K})$ & $\log g(\mathrm{dex})$ & {$[\mathrm{Fe} / \mathrm{H}](\mathrm{dex})$} & {$[\alpha / \mathrm{Fe}](\mathrm{dex})$} & $V_{r}\left(\mathrm{~km} \mathrm{~s}^{-1}\right)$ & $M_{\mathrm{V}}(\mathrm{mag})$ & $M_{\mathrm{Ks}}(\mathrm{mag})$ \\
\hline SNRs $>20$ & 150 & 0.2 & 0.15 & 0.1 & 5 & $0.5-0.6$ & $0.5-0.6$ \\
\hline SNRs $>50$ & 100 & 0.1 & 0.1 & 0.05 & 5 & $0.2-0.3$ & $0.2-0.3$ \\
\hline
\end{tabular}

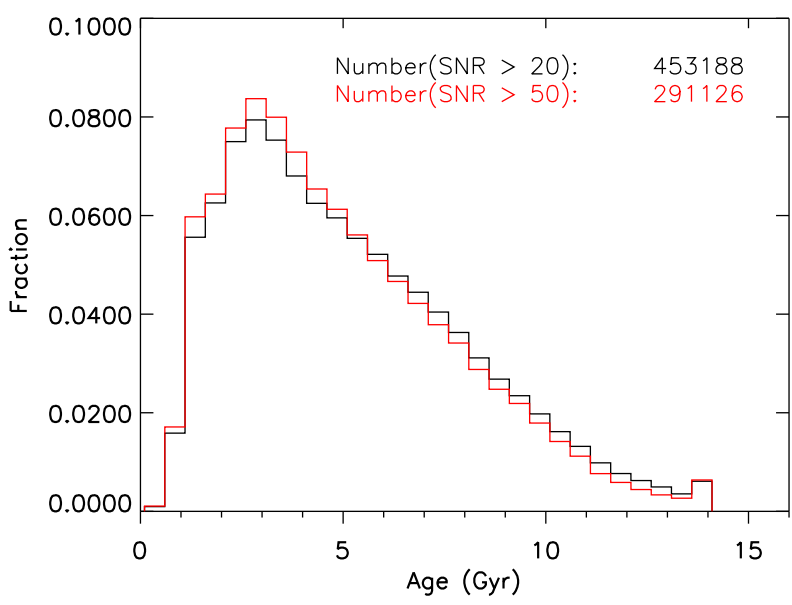

Figure 2. Normalised age distributions of the MSTO-SG-SNR20 (black line) and MSTO-SG-SNR50 (red line) samples. The total numbers of stars in the two samples are labeled in the Figure.

of the absolute magnitude, the limiting magnitude of the LAMOST Galactic spectroscopic surveys and the dust extinction. Considering the absolute magnitudes of the MSTO-SG stars vary significantly with age, we define a complete volume for each of the mono-age stellar populations of age range $0.0<\tau<2.0,2.0<\tau<4.0$, $4.0<\tau<6.0,6.0<\tau<8.0,8.0<\tau<11.0,11.0<\tau<14.0$ and $0<\tau<14.0 \mathrm{Gyr}$, respectively. Sample stars falling outside the defined complete volume are discarded. The final completed samples of MSTO-SG-SNR20 and MSTO-SG-SNR50 contain 453,188 and 291,126 stars, respectively.

Fig 1 shows the distributions of $[\mathrm{Fe} / \mathrm{H}],[\alpha / \mathrm{Fe}]$ and number density of the final (complete) MSTO-SG-SNR20 and MSTO-SGSNR50 samples in the $R-Z$ plane. Here $R$ and $Z$ are the axes of the Galactocentric cylindrical coordinate system $(R, \Phi, Z)$. The Sun is assumed to be at the Galactic disc mid-plane (i.e. $Z=0 \mathrm{pc}$ ) and has a $R$ value of $8 \mathrm{kpc}$. Fig 2 shows the normalised age distributions of the final MSTO-SG-SNR20 and MSTO-SG-SNR50 samples. The distributions show that for both samples, the stars have a broad distribution in stellar age, from $<1 \mathrm{Gyr}$ to $>13 \mathrm{Gyr}$, and the distributions peak at 3-4 Gyr.

\section{RADIAL AND VERTICAL GRADIENTS OF [FE/H] AND $[\alpha /$ FE $]$}

In this Section, we investigate the radial and vertical gradients of $[\mathrm{Fe} / \mathrm{H}]$ with the MSTO-SG-SNR20 sample and the radial and vertical gradients of $[\alpha / \mathrm{Fe}]$ with the MSTO-SG-SNR50 sample using a method similar to Xiang et al. (2015b) in order to better compare with their results.

\subsection{The radial $[\mathrm{Fe} / \mathrm{H}]$ gradients}

Firstly, we determine the radial $[\mathrm{Fe} / \mathrm{H}]$ gradients of mono-age stellar populations in several thin slices of $|Z|$. In doing so, we divide the stars in the MSTO-SG-SNR20 sample into mono-age populations of 6 age bins: $\tau<2.0,2.0<\tau<4.0,4.0<\tau<6.0$, $6.0<\tau<8.0,8.0<\tau<11.0,11.0<\tau<14.0$ Gyr. The division of age bins is the same as Xiang et al. (2015b), which allows a direct comparison with their results. Further we divide each of the mono-age populations into bins of a constant thickness of $\Delta|Z|=0.3 \mathrm{kpc}$ and $0.5 \mathrm{kpc}$ for stars of $|Z|<1.5 \mathrm{kpc}$ and $|Z|>1.5 \mathrm{kpc}$, respectively. In each age and height bin, we further divide the stars into small radial annuli of $0.25 \mathrm{kpc}$. Bins containing less than 30 stars are discarded. A straight line is then adopted to fit the CMD-weighted mean values of $[\mathrm{Fe} / \mathrm{H}]$ in the individual radial annuli, as a function of $R$. The slope of the line is adopted as the radial $[\mathrm{Fe} / \mathrm{H}]$ gradient. Fig 3 plots the results for two $|Z|$ slices of several individual mono-age stellar populations: $|Z|<0.3 \mathrm{kpc}$ and $1.5<|Z|<2.0 \mathrm{kpc}$. It shows that the CMD-weighted mean values of $[\mathrm{Fe} / \mathrm{H}]$ as a function of $R$ can be well fitted by a straight line. The dip in the number of stars at $R \sim 8 \mathrm{kpc}$ and $|Z|<0.3 \mathrm{kpc}$ in the Figure is a consequence of the bright limiting magnitude ( $\sim 9$ mag in $r$-band) of the survey.

$\mathrm{Fig} 4$ shows the derived radial $[\mathrm{Fe} / \mathrm{H}]$ gradients as a function of $|Z|$ for mono-age stellar populations. It shows that there are negative radial $[\mathrm{Fe} / \mathrm{H}]$ gradients for stellar populations of $\tau<11$ Gyr at $|Z|<1.5 \mathrm{kpc}$. The negative radial metallicity gradients flatten with increasing $|Z|$. The gradients of stellar populations of $\tau>11$ Gyr have marginally positive values except the results of $|Z|<0.3 \mathrm{kpc}\left(\sim-0.03 \pm 0.04 \mathrm{dex} \mathrm{kpc}^{-1}\right)$, and vary little with $|Z|$.

The values of radial $[\mathrm{Fe} / \mathrm{H}]$ gradients presented in the current paper are slightly larger than those of Xiang et al. (2015b) by 0.0 $-0.05 \mathrm{dex} \mathrm{kpc}^{-1}$. The differences are larger at low $|Z|$ bins. As discussed in Section 2.1, compared to Xiang et al. (2015b), ages, distances and atmospheric parameters of stars in the current sample have been improved. The current work also adopts different criteria of selecting MSTO-SG (or simply MSTO) compared to Xiang et al. (2015b). We re-estimate the radial $[\mathrm{Fe} / \mathrm{H}]$ gradients using the common stars of the current sample and that used by Xiang et al. (2015b) to check whether the difference of the parameters are mainly responsible for the difference. The exercise yields very similar results, suggesting that the differences in the derived parameters are not mainly responsible for the differences of the results of radial $[\mathrm{Fe} / \mathrm{H}]$ gradients derived in the current work and those presented in Xiang et al. (2015b). A further inspection of the MSTO sample of Xiang et al. (2015b) using the updated set of stellar parameters suggests that a considerable fraction of the sample stars are actually main-sequence star contaminations due to systematic errors in the (old version) LSP3 $\log g$ estimates, which are used by Xiang et al. (2015b) to select the MSTO sample stars. The contamination rate is a function of $T_{\text {eff }}$ in the sense that the cooler the stars, the higher the fraction of the contaminations. Because of the magnitude limits of the LAMOST surveys, the closer to the Sun, the cooler and more metal-rich stars could be observed by LAMOST as they are intrinsically fainter. Besides, most of the Galactic radii 

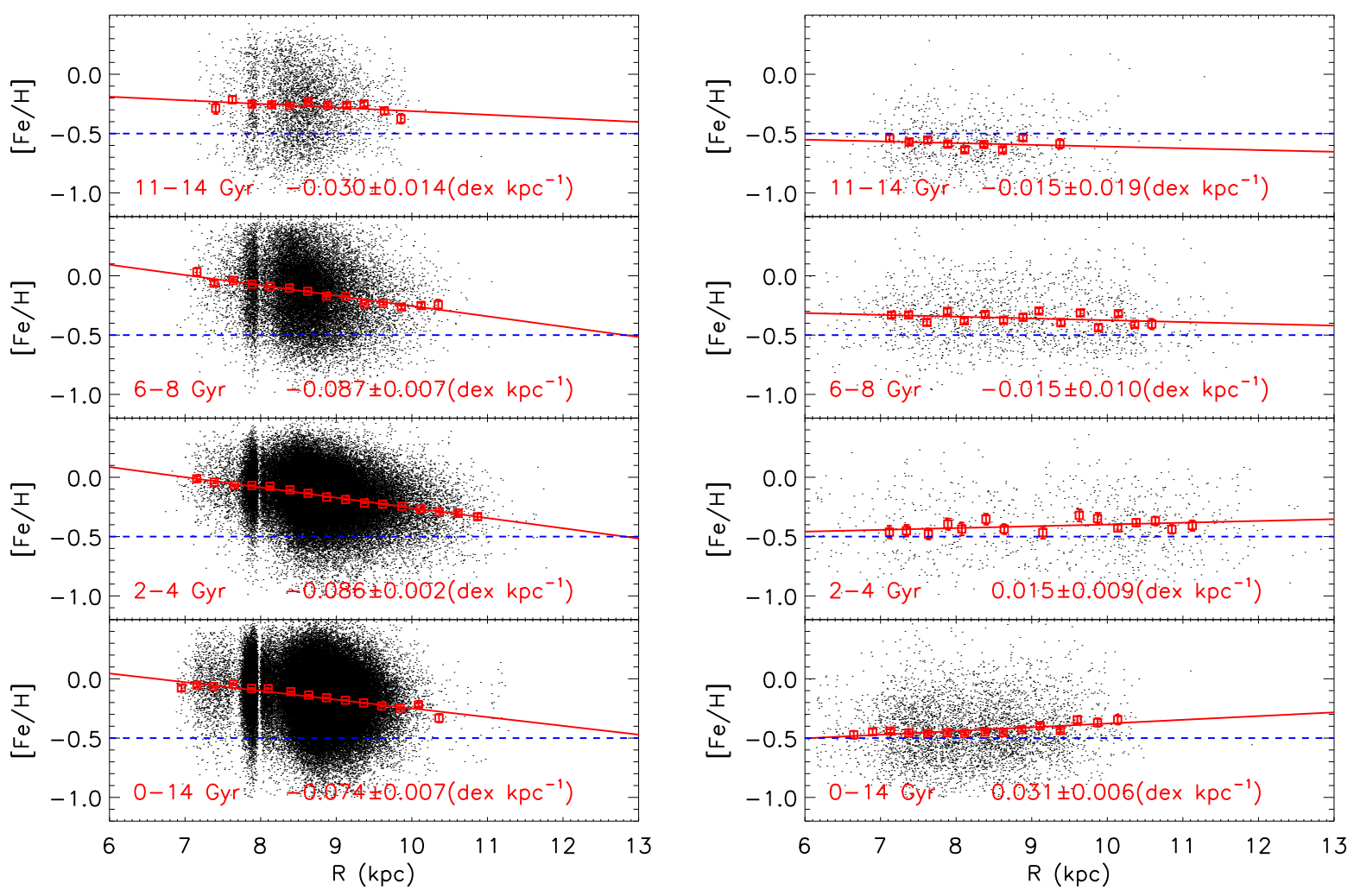

Figure 3. Radial $[\mathrm{Fe} / \mathrm{H}]$ gradients derived from several mono-age stellar populations (as marked in each panel) in two height slices: $|Z|<0.3 \mathrm{kpc}$ (left panels), $1.5<|Z|<2.0 \mathrm{kpc}$ (right panels). Red squares represent the CMD-weighted mean values of $[\mathrm{Fe} / \mathrm{H}]$ of the individual radial bins. The error bars of the mean values are also plotted, but in almost all the cases they are smaller than the size of the symbols. Linear fits to the red squares are over plotted by red lines. The blue horizontal dashed lines delineate a constant $[\mathrm{Fe} / \mathrm{H}]$ value of -0.5 dex. The age span of each population, the slope of the linear fit (the radial $[\mathrm{Fe} / \mathrm{H}]$ gradient) and the associated error, are marked in each panel. The mean value of $[\mathrm{Fe} / \mathrm{H}]$ as a function of $R$ in each panel can be well fitted by a linear line.

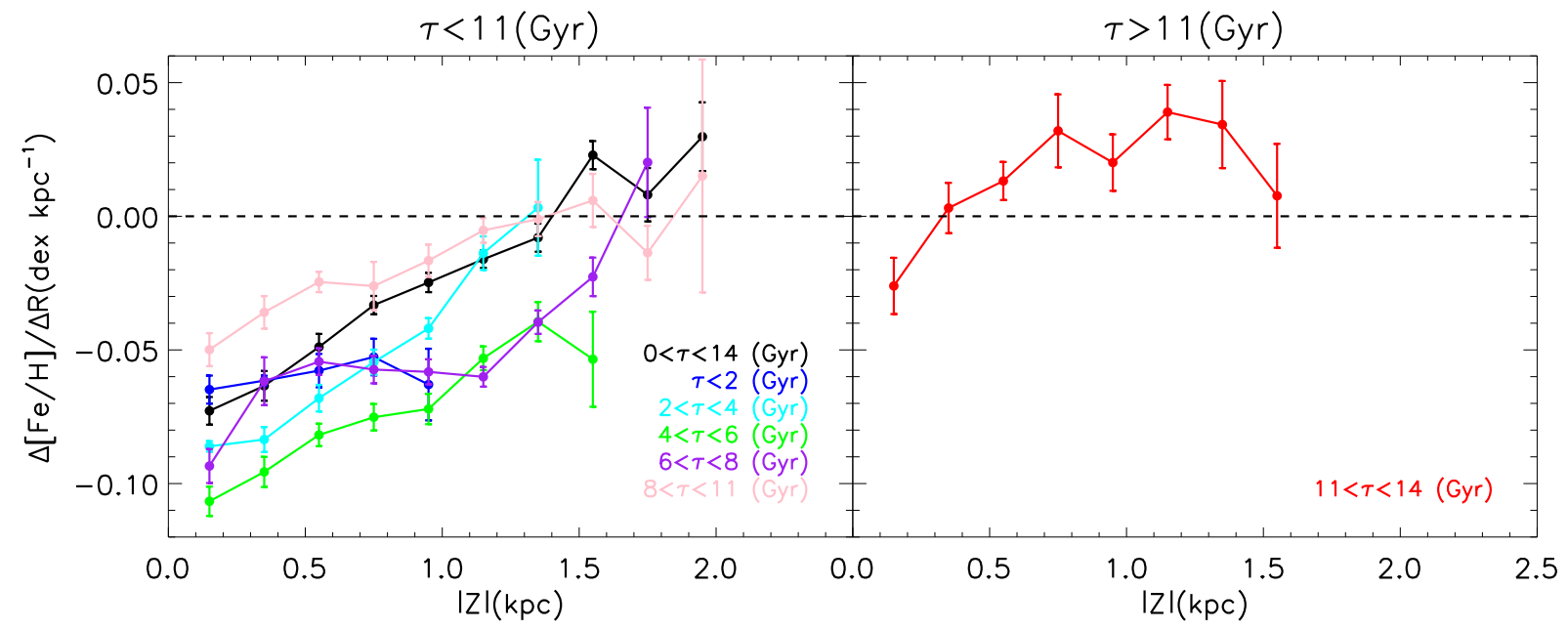

Figure 4. Radial $[\mathrm{Fe} / \mathrm{H}]$ gradients as a function of $|Z|$ derived from mono-age populations, presented with different colours, as marked in the plot. The horizontal dashed lines delineate a constant radial $[\mathrm{Fe} / \mathrm{H}]$ gradient value of $0.0 \mathrm{dex} \mathrm{kpc}^{-1}$. The mean radial $[\mathrm{Fe} / \mathrm{H}]$ gradients for the young $(\mathrm{left}$ panel) and the old (right panel) stellar populations are negative and positive, respectively. 


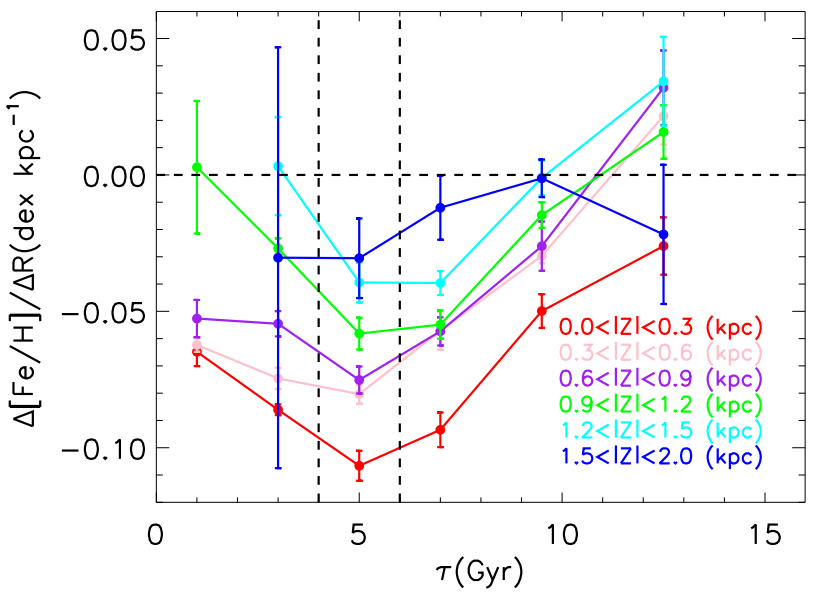

Figure 5. Radial $[\mathrm{Fe} / \mathrm{H}]$ gradients as a function of $\tau$ derived from stars at different $|Z|$ slices, presented with different colours, as marked in the plot. The horizontal dashed line delineates a constant radial $[\mathrm{Fe} / \mathrm{H}]$ gradient of $0.0 \mathrm{dex} \mathrm{kpc}^{-1}$. The vertical dashed lines delineate constant ages of 4 and 6 Gyr. There are dips in the radial $[\mathrm{Fe} / \mathrm{H}]$ gradients at $4<\tau<6 \mathrm{Gyr}$.

of the MSTO stars in Xiang et al. (2015b) are larger than $7.5 \mathrm{kpc}$ (see their Fig. 13), the radial gradients are derived with the stars of $R>7.5 \mathrm{kpc}$ (see their Fig. 14). Thus, we can approximately think that there are more contaminations (by more metal-rich stars) in the inner disc than in the outer disc, which produce the steeper radial $[\mathrm{Fe} / \mathrm{H}]$ gradients in Xiang et al. (2015b). Also, the contaminations of metal-rich stars are more severe at lower heights, which causes more overestimation (steeper) of radial $[\mathrm{Fe} / \mathrm{H}]$ gradients at lower disc. So that in conclusion, the differences of radial $[\mathrm{Fe} / \mathrm{H}]$ gradients between this work and Xiang et al. (2015b) are likely caused by overestimation of the results derived by Xiang et al. (2015b) due to non-negligible main-sequence star contaminations in their MSTO sample.

The vertical variations of radial $[\mathrm{Fe} / \mathrm{H}]$ gradients are quite similar to what found by Xiang et al. (2015b) as shown in their Fig. 15. Minchev, Chiappini \& Martig (2014) find that the negative radial metallicity gradients strongly flatten with increasing $|Z|$ in their chemodynamical models (see their Fig. 10). This is consistent with our results for stellar populations of $\tau<11 \mathrm{Gyr}$.

Fig 5 shows the derived radial $[\mathrm{Fe} / \mathrm{H}]$ gradients as a function of age for stars at different $|Z|$ bins. It shows that for stellar populations of $\tau<4 \mathrm{Gyr}$, the gradients steepen with increasing age, reaching a maximum at $4<\tau<6 \mathrm{Gyr}$, and then flatten with age.

The existence of reversions of radial $[\mathrm{Fe} / \mathrm{H}]$ gradients as a function of age are also found by Xiang et al. (2015b) (see their Fig. 16). However, they found the reversion occurs at $6<\tau<$ 8 Gyr. As we know the stellar age used by Xiang et al. (2015b) are systematically older than those deduced by Xiang et al.(2017a) for stars of $\tau>6 \mathrm{Gyr}$. This accounts for the difference of the reversion epoch. Interestingly, the epoch of reversion derived here is closer to that obtained by Casagrande et al. (2011) ( $\sim 4$ Gyr) for the solar-neighbourhood disc $(Z<100 \mathrm{pc})$ using GCS FG(K) dwarf stars, as shown in their Fig. 18. The results are not consistent with that obtained by the models of Toyouchi \& Chiba (2018), who find that the radial $[\mathrm{Fe} / \mathrm{H}]$ gradients monotonically increasing as age increases. The disagreement may be the response of their too simple and perhaps unrealistic assumptions for gas infall, gas outflow, gas reaccretion and the stellar radial migration processes, or lack of some important ingredients for an understanding of the MW formation (Toyouchi \& Chiba 2018).

\subsection{The vertical $[\mathrm{Fe} / \mathrm{H}]$ gradients}

To derive the vertical $[\mathrm{Fe} / \mathrm{H}]$ gradients, we divide the stars in the MSTO-SG-SNR20 sample into radial annuli of $1 \mathrm{kpc}$ with a step of $0.5 \mathrm{kpc}$ in the radial direction for the mono-age stellar populations. In each annulus, we further divide the stars into bins of constant height of $\Delta|Z|=0.2 \mathrm{kpc}$ with a step of $0.1 \mathrm{kpc}$. Bins containing less than 30 stars are discarded. The CMD-weighted mean values of $[\mathrm{Fe} / \mathrm{H}]$ in the individual $|Z|$ slices as a function of $|Z|$ are fitted by straight lines to derive the vertical $[\mathrm{Fe} / \mathrm{H}]$ gradients. Fig. 6plots the results for two radial bins of several mono-age stellar populations: $7<R<8 \mathrm{kpc}$ and $9<R<10 \mathrm{kpc}$. It shows that CMD-weighted mean values of $[\mathrm{Fe} / \mathrm{H}]$ as a function of $|Z|$ can be well fitted by a straight line. Fig. 6 shows that old stars reach much further above the disc than young stars. Few stars are sampled at $7<R<$ $8 \mathrm{kpc}$ and $|Z| \sim 0 \mathrm{kpc}$ because of the bright limiting magnitude of the surveys.

Fig 7 shows the derived vertical $[\mathrm{Fe} / \mathrm{H}]$ gradients as a function of $R$ for the mono-age stellar populations. It shows that almost all the mono-age stellar populations have negative vertical $[\mathrm{Fe} / \mathrm{H}]$ gradients. The vertical $[\mathrm{Fe} / \mathrm{H}]$ gradients flatten with increasing $R$ for all the mono-age stellar populations at $R>8 \mathrm{kpc}$. The vertical $[\mathrm{Fe} / \mathrm{H}]$ gradients vary significantly with age.

Compared to Xiang et al. (2015b), we have extended the measurement of vertical $[\mathrm{Fe} / \mathrm{H}]$ gradients toward the inner disc to $R<7 \mathrm{kpc}$ and found that the vertical $[\mathrm{Fe} / \mathrm{H}]$ gradients in the inner disc are much flatter than that in the solar neighbourhood. The vertical $[\mathrm{Fe} / \mathrm{H}]$ gradients derived in the current work are consistent with those obtained by Xiang et al. (2015b) for populations younger than $11 \mathrm{Gyr}$. The derived vertical $[\mathrm{Fe} / \mathrm{H}]$ gradients for the oldest populations ( $\tau>11 \mathrm{Gyr}$ ) are comparable to those for $8<\tau<11$ Gyr in the current work. Whereas Xiang et al. (2015b) found much shallower gradients for their oldest populations than those for $8<\tau<11$ Gyr. We believe that this is because Xiang et al. (2015b) discard stars cooler than 5,400 K. Such a temperature cut may have rejected some old metal-rich stars which are mostly found at low $|Z|$ 's, leading to shallower vertical $[\mathrm{Fe} / \mathrm{H}]$ gradients for the populations of $\tau>11 \mathrm{Gyr}$. Of course, it is also possible that those old metal-rich MSTO-SG stars in the current sample may be contaminations from younger dwarf stars. A further study is needed to deeper understand the results. The cause of the differences in the vertical $[\mathrm{Fe} / \mathrm{H}]$ gradients of the oldest stellar populations $(\tau>11$ Gyr) needs to be further explored.

In the current work, we do not find a monotonously increase of vertical $[\mathrm{Fe} / \mathrm{H}]$ gradient with age, in contrast to Xiang et al. (2015b. Fig. 17), who find that (with the exception of the oldest stars of $\tau>11 \mathrm{Gyr}$ ) the younger the stars, the flatter the vertical $[\mathrm{Fe} / \mathrm{H}]$ gradients. Ciucă et al. (2018, Fig. 6) also find a clear trend of vertical $[\mathrm{Fe} / \mathrm{H}]$ gradient with age using common dwarf stars selected from Gaia and RAVE, quite similar to what Xiang et al. (2015b) find. We note that the observational selection bias of the samples of Ciucǎ et al. (2018) and Xiang et al. (2015b) are not removed completely. Ciucă et al. (2018) only minimise the observational selection bias induced by the various selection criteria by selecting a sample in a specific colour and magnitude range. Xiang et al. (2015b) did not consider the effects of incomplete volume of monoage stellar populations on the derived vertical $[\mathrm{Fe} / \mathrm{H}]$ gradients. The selection bias may be responsible for the difference of the trend be- 

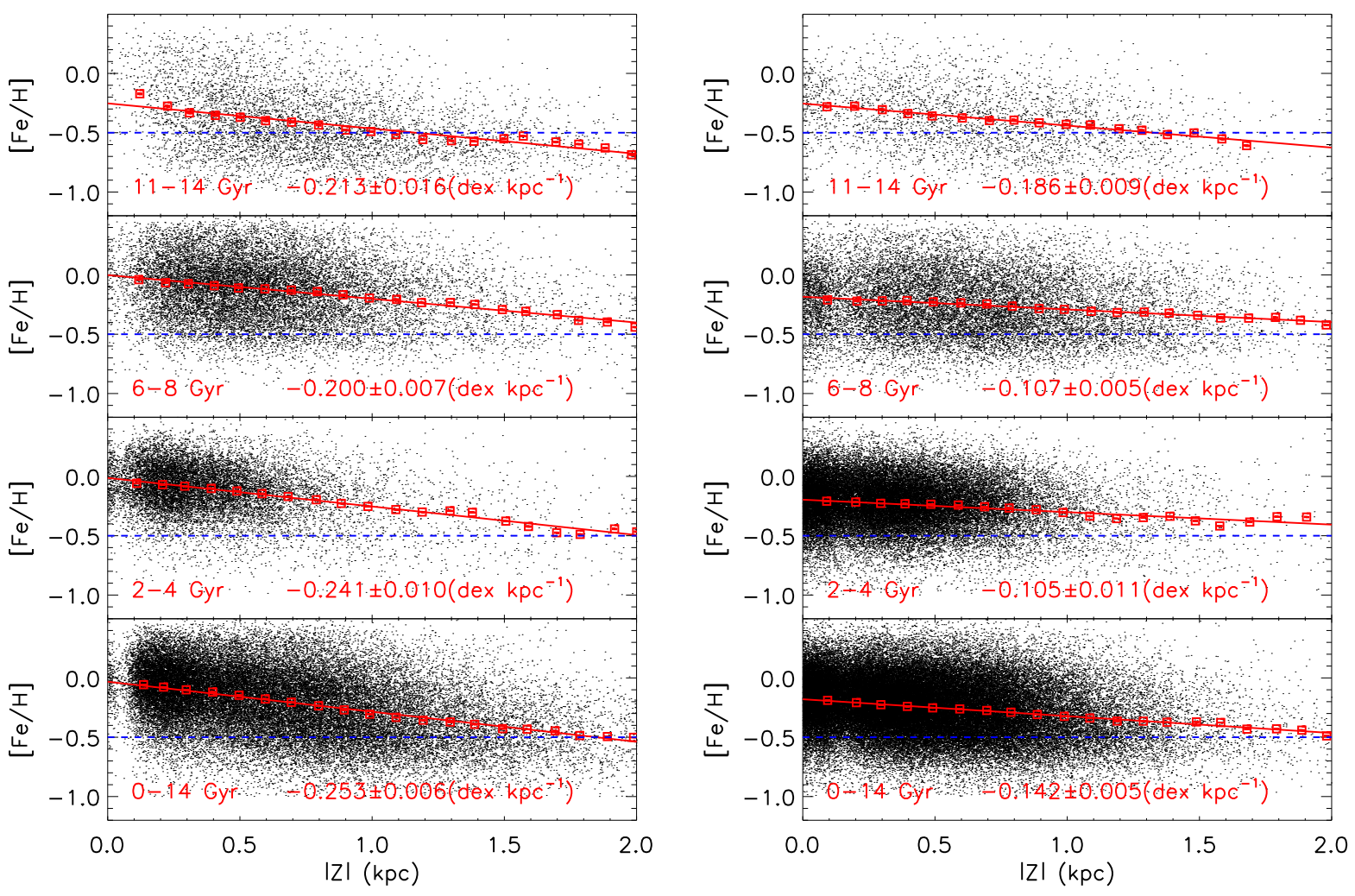

Figure 6. Vertical $[\mathrm{Fe} / \mathrm{H}]$ gradients derived for several individual mono-age stellar populations (as marked in each panel) in two radial bins: $7<R<8 \mathrm{kpc}$ (left panels) and $9<R<10 \mathrm{kpc}$ (right panels). Red squares represent the CMD-weighted mean values of [Fe/H] in the individual vertical bins. The error bars of the mean values are also over plotted, but in almost all the cases they are smaller than the size of the symbols. Linear fits to red squares are over plotted by red lines. The blue horizontal dashed lines delineate a constant $[\mathrm{Fe} / \mathrm{H}]$ value of $-0.5 \mathrm{dex}$. The age range of each mono-age stellar population, the slope of the linear fit (vertical $[\mathrm{Fe} / \mathrm{H}]$ gradients) and the associated error are marked in each panel. The mean value of $[\mathrm{Fe} / \mathrm{H}]$ as a function of $|Z|$ in each panel can be well fitted by a straight line.

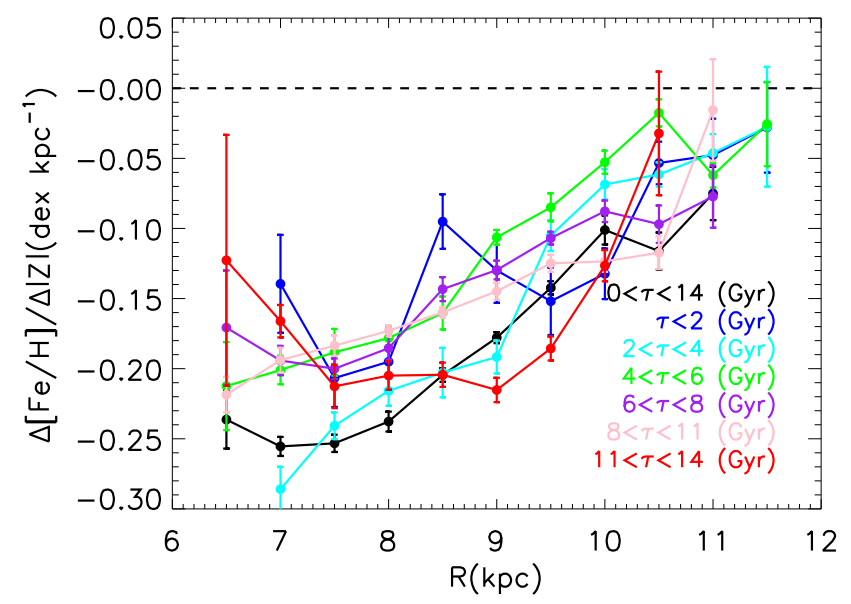

Figure 7. Vertical $[\mathrm{Fe} / \mathrm{H}]$ gradients as a function of $R$ derived from monoage stellar populations, presented with different colours, as marked in the plot. The horizontal dashed line delineates a constant vertical $[\mathrm{Fe} / \mathrm{H}]$ gradient of $0.0 \mathrm{dex} \mathrm{kpc}^{-1}$. Stars in all $R$ and $\tau$ bins have negative vertical $[\mathrm{Fe} / \mathrm{H}]$ gradients. tween our results and that of Ciucǎ et al. (2018) and Xiang et al. (2015b).

\subsection{The radial $[\alpha / \mathrm{Fe}]$ gradients}

We determine the radial $[\alpha / \mathrm{Fe}]$ gradients in thin $|Z|$ slices. In doing so, the stars in the MSTO-SG-SNR50 sample are divided into different $|Z|$ and age bins as described in Section 3.1. In each bin, stars are further divided into small radial bins of size of $0.25 \mathrm{kpc}$. Bins containing less than 30 stars are again discarded. The CMDweighted mean values of $[\alpha / \mathrm{Fe}]$ in the individual radial bins as a function of $R$ are fitted by a straight line to derive the radial $[\alpha / \mathrm{Fe}]$ gradient. As an example, Fig. 8 presents the results for several mono-age populations at the two $|Z|$ slices: $|Z|<0.3 \mathrm{kpc}$ and $1.5<|Z|<2.0 \mathrm{kpc}$. It shows that straight lines fit the trends well for almost all the mono-age stellar populations of almost all the $|Z|$ bins. An exception is for young stellar populations ( $\tau<4 \mathrm{Gyr}$ ), and this may be a consequence of poor $[\alpha / \mathrm{Fe}]$ estimates for those stars (Xiang et al. 2017a). For the population of all ages $(0<\tau<14 \mathrm{Gyr})$, the fits are also not very good, and this may be the consequence of the dominations of young disc stars with incorrect $[\alpha / \mathrm{Fe}]$ values at low $|Z|$ 's of the inner disc.

Fig 9 shows the derived radial $[\alpha / \mathrm{Fe}]$ gradients for the mono- 

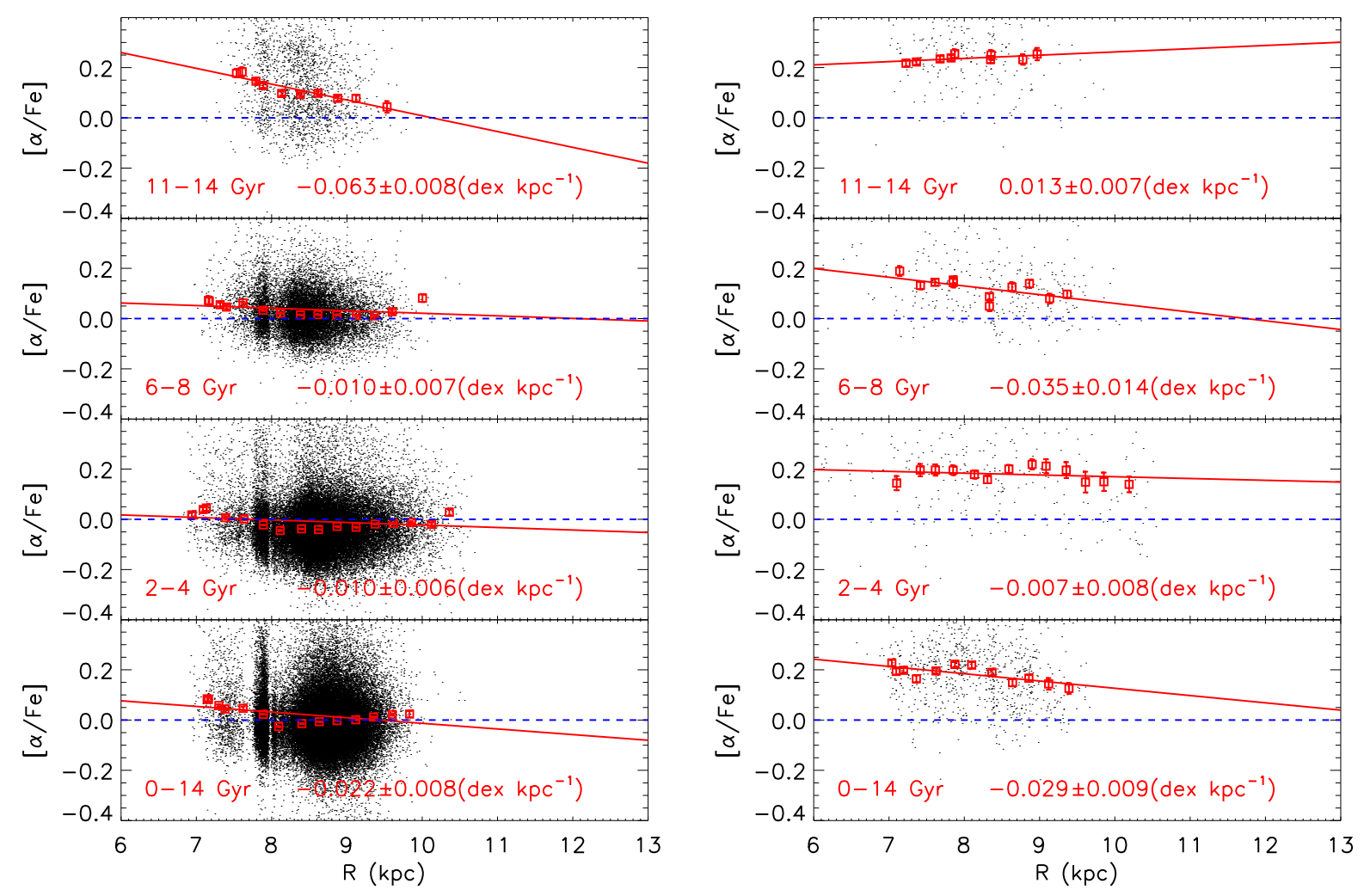

Figure 8. Radial $[\alpha / \mathrm{Fe}]$ gradients derived from several mono-age stellar populations (as marked in each panel) in two height slices: $|Z|<0.3 \mathrm{kpc}$ (left panels) and $1.5<|Z|<2.0 \mathrm{kpc}$ (right panels). Red squares represent the CMD-weighted mean values of $[\alpha / \mathrm{Fe}]$ of the individual radial bins. The error bars of the mean values are also plotted, but in almost all the cases they are smaller than the size of the symbols. Linear fits to the red squares are over plotted by red lines. The blue horizontal dashed lines delineate a constant $[\alpha / \mathrm{Fe}]$ value of 0.0 dex. The age span of each population, the slope of the linear fit (the radial $[\alpha / \mathrm{Fe}]$ gradient) and the associated error, are marked in each panel. In some $|Z|$ and $\tau$ bins, $[\alpha / \mathrm{Fe}]$ as a function of $R$ can not be well fitted by a linear line.

age stellar populations as a function of $|Z|$. The current work investigate the radial $[\alpha / \mathrm{Fe}]$ gradients of mono-age stellar populations for the first time and find that the radial $[\alpha / \mathrm{Fe}]$ gradients and its variations with $|Z|$ vary significantly with age. Fig 9 shows that the radial $[\alpha / \mathrm{Fe}]$ gradients of population of all ages are nearly flat at low $|Z|$ 's, and negative at high $|Z|$ 's. The radial $[\alpha / \mathrm{Fe}]$ gradients of populations of $\tau<4 \mathrm{Gyr}$ have positive values at low $|Z|$ 's, and negative at high $|Z|$ 's, and become smaller as $|Z|$ increases. Stars of ages $4<\tau<6 \mathrm{Gyr}$ have nearly flat radial $[\alpha / \mathrm{Fe}]$ gradients. Stars older than $\tau>6$ Gyr have negative radial $[\alpha / \mathrm{Fe}]$ gradients. The radial $[\alpha / \mathrm{Fe}]$ gradients of old stellar populations $(\tau>8 \mathrm{Gyr})$ flatten as $|Z|$ increases.

Several previous works have studied the radial $[\alpha / \mathrm{Fe}]$ gradients of the population of all ages. Anders et al. (2014) show that there are almost flat radial $[\alpha / \mathrm{Fe}]$ gradients at $Z_{\max }<0.4 \mathrm{kpc}$, but clear negative $[\alpha / \mathrm{Fe}]$ gradients at $1.5<Z_{\max }<3.0 \mathrm{kpc}$, derived from an analysis of APOGEE red giant stars as shown in their Fig. 16. Minchev, Chiappini \& Martig (2014) have also found that the weak positive $[\mathrm{Mg} / \mathrm{Fe}]$ gradient for the all-age stellar population turns negative as $|Z|$ increases (see their Fig. 10). Boeche et al. (2014) have found that the radial $[\mathrm{Mg} / \mathrm{Fe}],[\mathrm{Al} / \mathrm{Fe}]$ and $[\mathrm{Si} / \mathrm{Fe}]$ gradients are positive at low $|Z|$ 's, and negative at high $|Z|$ 's using giant stars selected from RAVE (see their Table 2). Our current result of the stellar population of all ages are consistent with those of
Anders et al. (2014) and Minchev, Chiappini \& Martig (2014), but are slightly different compared to those by Boeche et al. (2014).

Fig 10 shows the derived radial $[\alpha / \mathrm{Fe}]$ gradients as a function of age for stars at different $|Z|$ 's. It shows that the derived negative radial $[\alpha / \mathrm{Fe}]$ gradients steepen with increasing age at low $|Z|$ 's, but flatten or become nearly invariant with increasing age at high $|Z|$ 's. Minchev, Chiappini \& Martig (2014) have found that there is a nearly flat $[\mathrm{Mg} / \mathrm{Fe}]$ gradient for old stellar populations, but a positive radial $[\mathrm{Mg} / \mathrm{Fe}]$ gradient for young stellar population (see their Fig. 10) in their chemodynamical models, some different from what found here. Our results may strongly constrain the existing chemodynamical models.

\subsection{The vertical $[\alpha / \mathrm{Fe}]$ gradients}

We now determine the vertical $[\alpha / \mathrm{Fe}]$ gradients. In doing so, we divide the stars in the MSTO-SG-SNR50 sample into annuli of 1.0 $\mathrm{kpc}$ with a step of $0.5 \mathrm{kpc}$ in the radial direction for the mono-age stellar populations. In each annulus, we further divide the stars into bins of a constant thickness of $\Delta|Z|=0.2 \mathrm{kpc}$ with a step of $0.1 \mathrm{kpc}$. Bins containing less than 30 stars are discarded. For each annulus, the CMD-weighted mean values of $[\alpha / \mathrm{Fe}]$ are estimated for all height slices. The results as a function of $|Z|$ are fitted by a straight line. The slope of the straight line is adopted as the vertical 
gradient of the mono-age stellar population at that radial annulus. Fig 11 shows the results at two radial bins: $7<R<8 \mathrm{kpc}$ and $9<R<10 \mathrm{kpc}$. It shows that the CMD-weighted mean values of $[\alpha / \mathrm{Fe}]$ as a function of $|Z|$ are well fitted by a linear line.

Fig 12 shows the vertical $[\alpha / \mathrm{Fe}]$ gradients derived for the mono-age stellar populations as a function of $R$. Almost all of the mono-age stellar populations have positive vertical $[\alpha / \mathrm{Fe}]$ gradients at a given radial annulus. It also shows that the positive vertical $[\alpha / \mathrm{Fe}]$ gradients flatten with increasing $R$ for the stellar populations of $\tau<8 \mathrm{Gyr}$. The gradients steepen with increasing $R$ for the stellar populations of $\tau>8 \mathrm{Gyr}$.

\subsection{Uncertainties of the gradients}

Throughout the paper, only the fitting errors are reported, and the main contribution are random errors of the stellar parameters ([Fe/H], $R, Z$ and age). In most cases, the fitting errors are very small $\left(<0.01 \mathrm{dex} \mathrm{kpc}^{-1}\right)$ because of the large number of stars in our sample. We emphasize that those small values of gradient errors are not in conflict with the broad metallicity distribution of stars at a given $R$ or $Z$. This is because the latter is not only contributed by random errors of stellar parameters but also largely contributed by the intrinsic width of metallicity distribution. However, there are indeed other error (systematic) sources that are not considered in this work. For example, we derive the gradients with linear functions, but which may fail to describe the true radial or vertical trend of mean $[\mathrm{Fe} / \mathrm{H}]$ or $[\alpha / \mathrm{Fe}]$ accurately. This is especially obvious in the case of the radial $[\alpha / \mathrm{Fe}]$ gradients (see Fig. 8). In addition, contaminations from dwarf stars to our current MSTO-SG star sample are also expected to lead considerable systematic errors to the gradients of old stellar populations.

\section{PROFILES OF THE [FE/H $]$ AND $[\alpha /$ FE $]$ DISTRIBUTIONS}

\subsection{Profiles of the $[\mathrm{Fe} / \mathrm{H}]$ distributions}

In order to investigate the profiles of the $[\mathrm{Fe} / \mathrm{H}]$ distributions, we firstly map out the $[\mathrm{Fe} / \mathrm{H}]$ distributions of mono-age stellar populations in each $R$ and $|Z|$ bin. In doing so, the individual mono-age stellar populations are divided into annuli of $1 \mathrm{kpc}$ and $3 \mathrm{kpc}$ in the radial direction for $R<12 \mathrm{kpc}$ and $R>12 \mathrm{kpc}$, respectively. In each annulus, we further divide the mono-age stellar populations into three bins of $|Z|:[0,0.3],[0.3,1.5]$ and $[1.5,2] \mathrm{kpc}$, corresponding roughly to the thin disc, the transition region between the thin and thick disc and the thick disc, respectively. Bins containing less than 100 stars are discarded. Fig 13 shows the normalised $[\mathrm{Fe} / \mathrm{H}]$ distributions of the individual mono-age stellar populations in each $R$ and $|Z|$ bin. In each bin, the histogram $[\mathrm{Fe} / \mathrm{H}]$ distribution is plotted with a bin size of $0.08 \mathrm{dex}$, corresponding approximately to half of the typical uncertainty of $[\mathrm{Fe} / \mathrm{H}]$ measurements. The profiles of the $[\mathrm{Fe} / \mathrm{H}]$ distributions of the different mono-age stellar populations show significant variations with $R$ and $|Z|$, especially for the stellar populations of $\tau<8 \mathrm{Gyr}$. For the stellar populations of $\tau<8 \mathrm{Gyr}$ and $|Z|<1.5 \mathrm{kpc}$, the [Fe/H] distributions vary from a negative skewed profile (having relative long metal-poor tails) at the inner disc to a positive skewed profile (having relative long metal-rich tails) as $R$ at the outer disc. For the stellar populations older than $8 \mathrm{Gyr}$ at all $|Z|$ bins, or the stellar populations younger than $8 \mathrm{Gyr}$ at high $|Z|$ bins, the distributions show only weak variations with $R$. For the stellar population of $\tau>11 \mathrm{Gyr}$, our results also show an unexpectedly high fraction of metal-rich $[\mathrm{Fe} / \mathrm{H}]>0$ stars, whose origins are not fully understood. A possibility is that they are actually originated at the inner disc, and migrated to their current position due to kinematic processes. Contaminations of younger dwarf stars may have also contributed a significant part of them.

In order to quantitatively describe the differences of the profiles of the $[\mathrm{Fe} / \mathrm{H}]$ distributions of the the individual mono-age stellar populations, we further derive the skewness of the distributions. In doing so, the individual mono-age stellar populations are divided into annuli of $1 \mathrm{kpc}$ in the radial direction from $6 \mathrm{kpc}$ to $13 \mathrm{kpc}$ with a step of $0.5 \mathrm{kpc}$. The bins of $|Z|$ are the same as used for Fig 13 Bins containing less than 600 stars are discarded to ensure robust measurements of the skewness. Fig 14 plots the radial variations of the skewness for mono-age stellar populations at $|Z|<1.5 \mathrm{kpc}$. The Figure shows a strong radial trend of the skewness, and the trend varies with stellar age. It shows that for populations of $\tau<8$ Gyr and $|Z|<1.5 \mathrm{kpc}$, the skewness increases with increasing $R$ (switchs from negative to flat even positive), and the radial trend becomes flatter with increasing age. For the old populations of $\tau>8 \mathrm{Gyr}$, the skewness decreases with increasing $R$. While the radial trend of the skewness of $8<\tau<11 \mathrm{Gyr}$ is less significant.

Hayden et al. (2015) have studied the radial variations of the profiles of the $[\mathrm{Fe} / \mathrm{H}]$ distributions (see their Fig. 5) using 69,919 APOGEE red-giant stars. In their study they divide the sample stars into two populations of high and low $[\alpha / \mathrm{Fe}]$ ratios, corresponding approximately to the old and young stellar populations, respectively. Their results show that the $[\mathrm{Fe} / \mathrm{H}]$ distributions of the low$[\alpha / \mathrm{Fe}]$ population close to the Galactic plane $(|Z|<0.5 \mathrm{kpc})$ are negatively skewed in the inner disc and are positively skewed in the outer disc. Whereas the profiles of $[\mathrm{Fe} / \mathrm{H}]$ distributions of the high- $[\alpha / \mathrm{Fe}]$ population, as well as those of the low- $[\alpha / \mathrm{Fe}]$ population at large height above the disc mid-plane, are almost symmetric and invariant with $R$. These radial variations trends of the profiles of $[\mathrm{Fe} / \mathrm{H}]$ distributions for low- $[\alpha / \mathrm{Fe}]$ and high- $[\alpha / \mathrm{Fe}]$ are generally in good agreement with our results for young $(<8 \mathrm{Gyr})$ and old ( $>8 \mathrm{Gyr}$ ) stellar populations, respectively. Benefiting from the robust age estimates, we are able to determine the transition age when the profiles of the $[\mathrm{Fe} / \mathrm{H}]$ distributions (more precisely, the skewness) change from the situation of strong radial variations (at $\tau<8 \mathrm{Gyr}$ ) to a situation of weak radial variations (at $\tau>8 \mathrm{Gyr}$ ).

\subsection{Profiles of the $[\alpha / \mathrm{Fe}]$ distributions}

To study the profiles of the $[\alpha / \mathrm{Fe}]$ distributions of the mono-age stellar populations, the MSTO-SG-SNR50 sample is divided into bins of $R,|Z|$ and $\tau$. The bins used in terms of $R,|Z|$ and $\tau$ are same as those adopted for Fig 13 The normalised $[\alpha / \mathrm{Fe}]$ distributions are shown on Fig 15 It shows that the profiles of the $[\alpha / \mathrm{Fe}]$ distributions vary with $R$ at all $|Z|$ 's for the populations of $\tau>8$ Gyr. They have negatively skewed distributions in the inner disc and positively skewed distributions in the outer disc. For the stellar populations of $2<\tau<8 \mathrm{Gyr}$, the profiles of the $[\alpha / \mathrm{Fe}]$ distributions vary only weakly with $R$ and $|Z|$. The results also show that the stellar population of $4<\tau<6 \mathrm{Gyr}$ at $R<7 \mathrm{kpc}$ contain much more $\alpha$-rich stars than at $R>7 \mathrm{kpc}$. Considering the small number of stars in this bin, we presume this result is not significant. The profiles of $[\alpha / \mathrm{Fe}]$ distributions of stellar populations of $\tau<2$ Gyr show variations with $R$. However, we note that the stellar populations of $\tau<2 \mathrm{Gyr}$ contains an unexpectedly high fraction of high $[\alpha / \mathrm{Fe}]$ stars, and this may be the consequence of incorrect $[\alpha / \mathrm{Fe}]$ estimates of some of those stars. As discussed by 

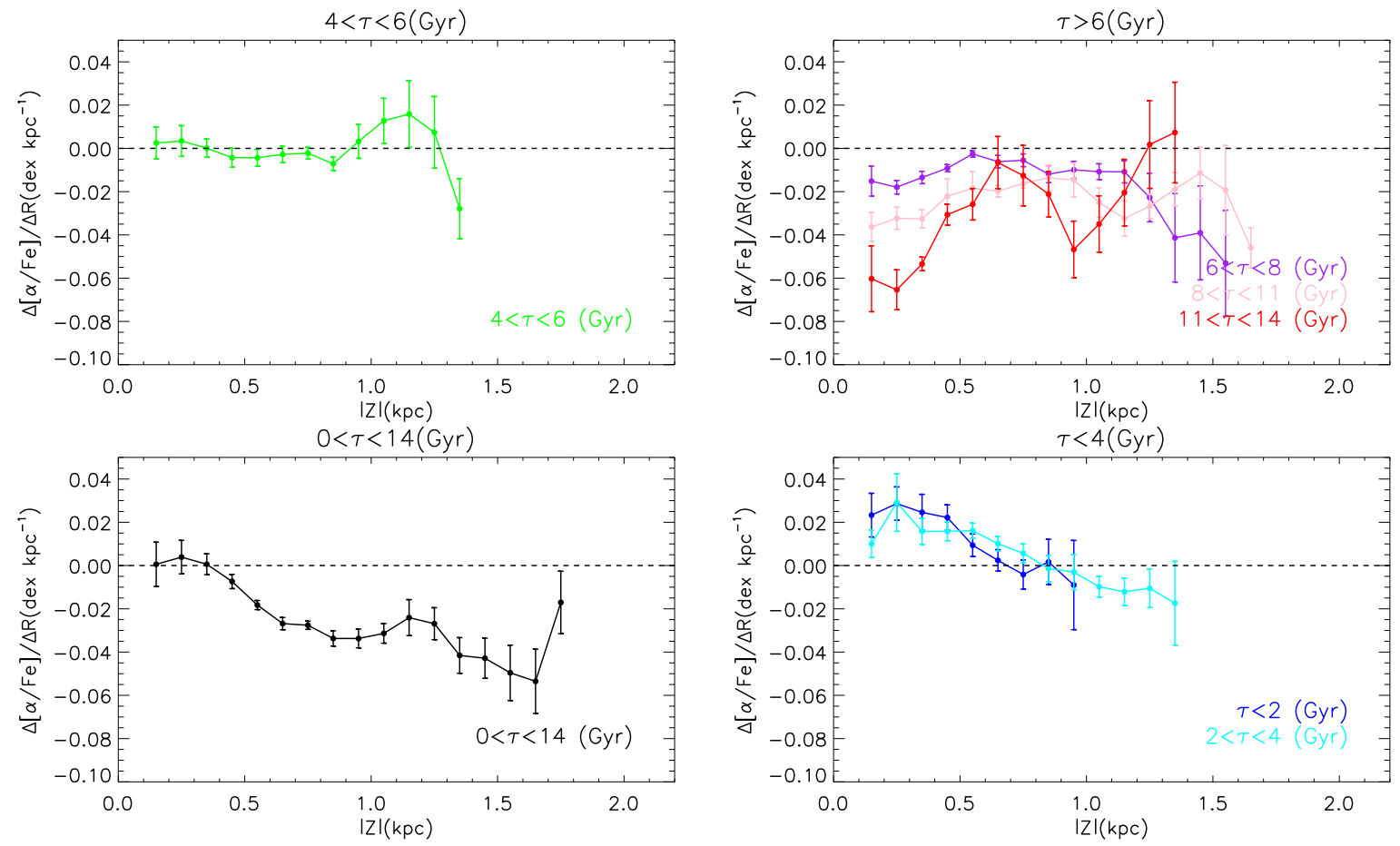

Figure 9. Radial $[\alpha / \mathrm{Fe}]$ gradients as a function of $|Z|$ derived from mono-age populations, presented with different colours, as marked in the plot. The mean radial $[\alpha / \mathrm{Fe}]$ gradients of all ages, young, median age and old stellar populations are negative, positive, near-zero and negative, respectively. The horizontal dashed lines delineate a constant radial $[\alpha / \mathrm{Fe}]$ gradient value of $0.0 \mathrm{dex} \mathrm{kpc}-1$. The results of young stellar populations should be taken with caution as about $10-20$ per cent $[\alpha / \mathrm{Fe}]$ measurements of those stars might be incorrect.

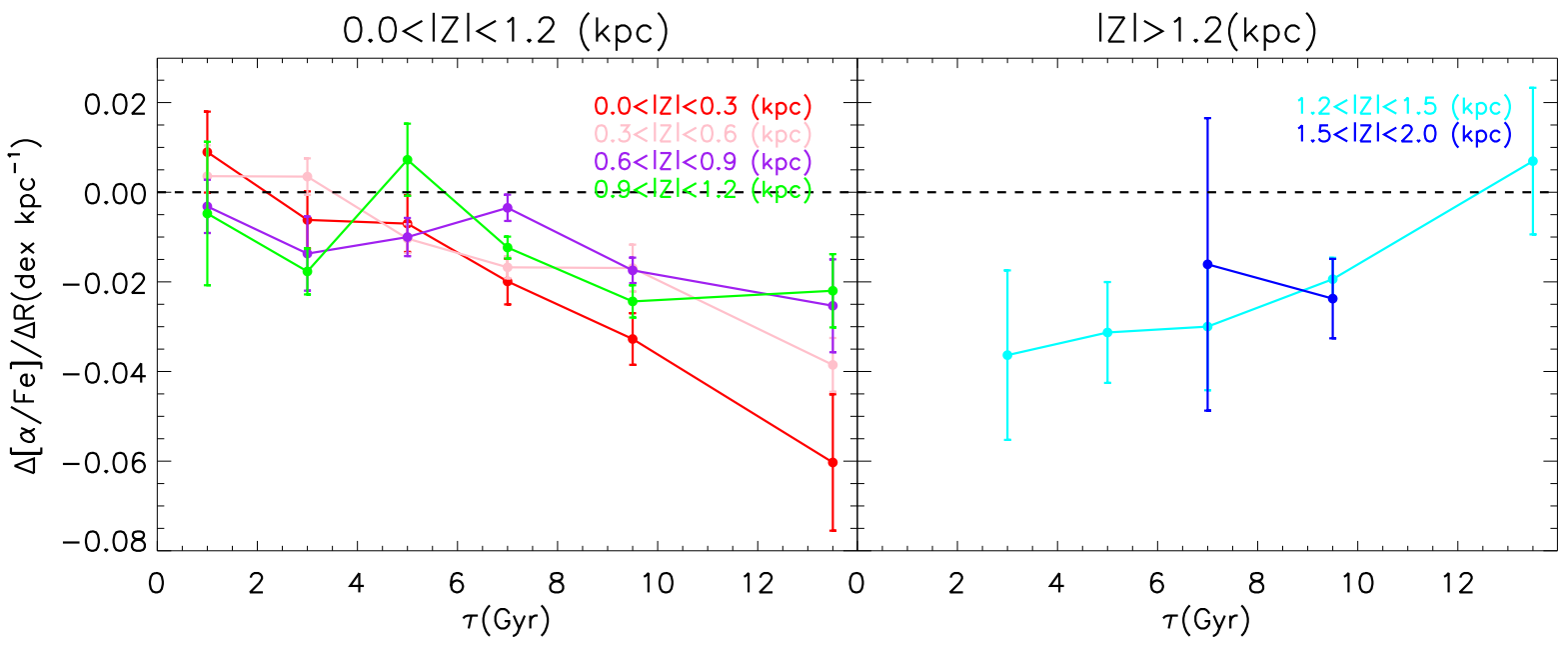

Figure 10. Radial $[\alpha / \mathrm{Fe}]$ gradients as a function of $\tau$ derived for stars at different $|Z|$ slices, presented with different colours, as marked in the plot. The horizontal dashed lines delineate a constant radial $[\alpha / \mathrm{Fe}]$ gradient value of $0.0 \mathrm{dex} \mathrm{kpc}{ }^{-1}$. Radial $[\alpha / \mathrm{Fe}]$ gradients steepen with increasing age at low $|Z|$ bins (left panel), but flatten or become nearly invariant with increasing age at high $|Z|$ bins (right panel).

Xiang et al. (2017a), those objects are likely spectroscopic binaries of composite spectra. The $[\alpha / \mathrm{Fe}]$ values of those stars have been incorrectly estimated. The fraction of youngest stars with incorrect values of $[\alpha / \mathrm{Fe}]$ is about $10-20$ per cent. Thus, the profiles of $[\alpha / \mathrm{Fe}]$ distributions of youngest stars should be taken with caution.

Values of skewness of the $[\alpha / \mathrm{Fe}]$ distributions are also esti- mated for the mono-age stellar populations, as shown in Fig 16 As discussed in the above paragraph, we find that the profiles of $[\alpha / \mathrm{Fe}]$ distributions of $\tau<2.0 \mathrm{Gyr}$ are likely to be unrealistic and that of $2<\tau<8$ Gyr vary weakly with $R$. Thus, values of skewness of the $[\alpha / \mathrm{Fe}]$ distributions of the young stellar populations are not presented here. Fig 16 shows only the radial variations of the skew- 

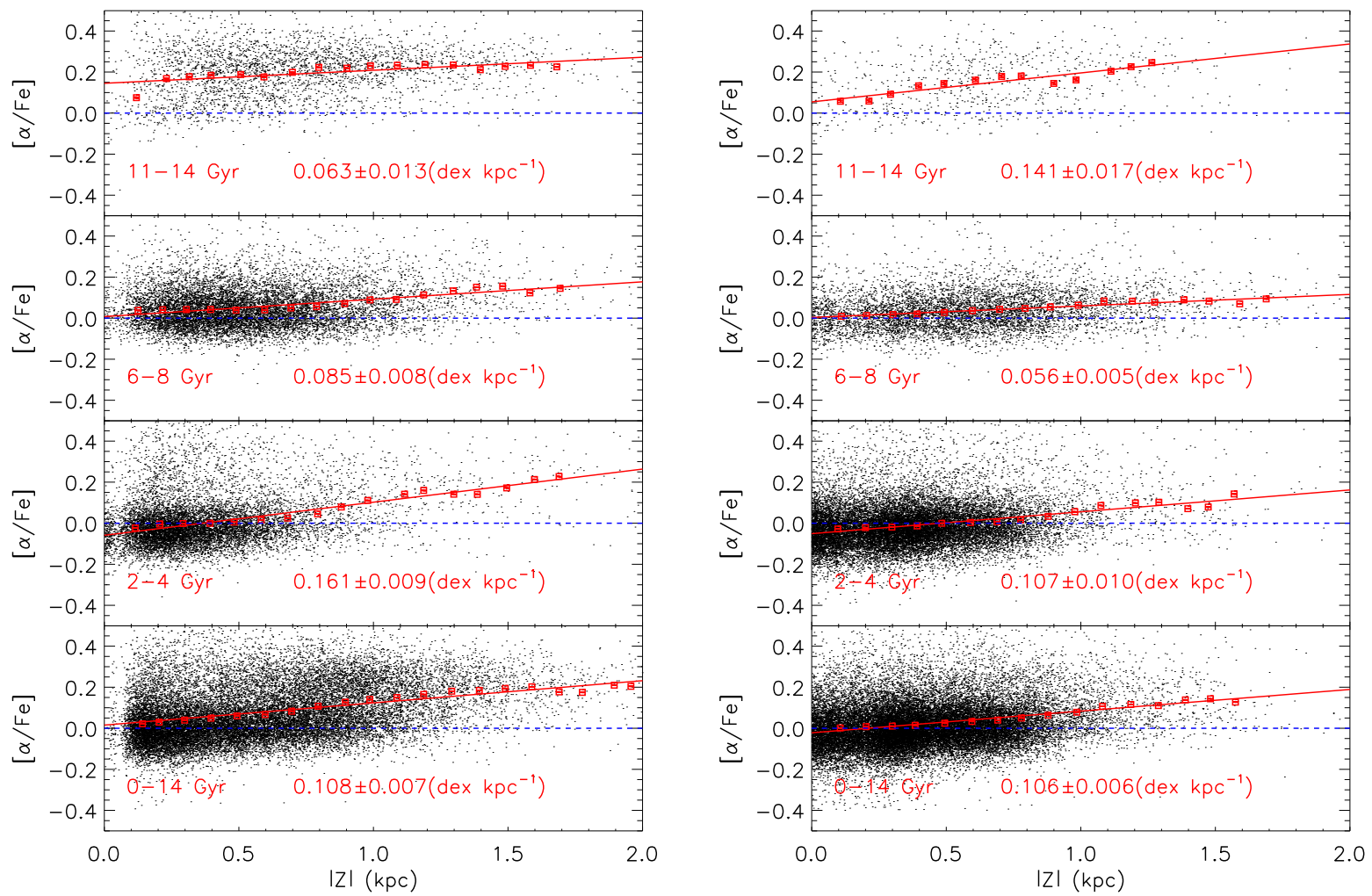

Figure 11. Vertical $[\alpha / \mathrm{Fe}]$ gradients derived from several individual mono-age stellar populations (as marked in each panel) in two radial bins: $7<R<8$ kpc (left panels) and $9<R<10 \mathrm{kpc}$ (right panels). Red squares represent the CMD-weighted mean values of $[\alpha / \mathrm{Fe}]$ in the individual vertical bins. The error bars of the mean values are also over plotted, but in almost all the cases they are smaller than the size of the symbols. Linear fits to red squares are over plotted by red lines. The blue horizontal dashed lines delineate a constant $[\alpha / \mathrm{Fe}]$ value of $0.0 \mathrm{dex}$. The age range of each mono-age stellar population, the slope of the linear fit (vertical $[\alpha / \mathrm{Fe}]$ gradients) and the associated error are marked in each panel. The mean value of $[\alpha / \mathrm{Fe}]$ as a function of $|Z|$ in each panel can be well fitted by a linear line.

ness of the $[\alpha / \mathrm{Fe}]$ distributions for the stellar populations of $\tau>8$ Gyr in different $|Z|$ bins. It shows that the skewness of the stellar populations of $\tau>8$ Gyr increase with increasing $R$ at $|Z|<1.5$ kpc.

\section{IMPACTS OF RADIAL MIGRATION ON THE METALLICITY DISTRIBUTION FUNCTION}

The radial dependence of the profiles of the $[\mathrm{Fe} / \mathrm{H}]$ distributions has been modelled by Schönrich \& Binney (2009) and Toyouchi \& Chiba (2018) using a chemical evolution model including the radial migration processes. The radial migration processes carry stars from the inner (metal-rich) or outer (metal-poor) disc region to their current spatial position $(R, Z)$, and consequently change the profiles of the $[\mathrm{Fe} / \mathrm{H}]$ distributions owing to the existence of radial metallicity gradients of the Galactic disc. Because the Galactic disc exhibits negative radial $[\mathrm{Fe} / \mathrm{H}]$ gradients, the inwards migrators generally contribute the metal-poor tail of the $[\mathrm{Fe} / \mathrm{H}]$ distribution, while the outwards migrators contribute mainly the metal-rich tail. The process will produce a relatively negative skewed $[\mathrm{Fe} / \mathrm{H}]$ distribution in the inner disc and a relatively positive skewed $[\mathrm{Fe} / \mathrm{H}]$ distribution in the outer disc, and consequently produce a positive radial gradient of skewness. That is the radial gradients of skewness and mean values and their evolution with age should be opposite in sign. Fig. 17 shows the radial gradients of skewness and mean values of $[\mathrm{Fe} / \mathrm{H}]$ and $[\alpha / \mathrm{Fe}]$ as a function of age for stellar populations at $|Z|<1.5 \mathrm{kpc}$. It shows that the radial gradients of skewness and mean values and their evolution with age are indeed opposite in sign, and the stronger the abundance gradients, the stronger the gradients in skewness. The radial $[\mathrm{Fe} / \mathrm{H}]$ gradients of stellar populations of $\tau<8 \mathrm{Gyr}$ at $|Z|<1.5 \mathrm{kpc}$ are negative, while the radial gradients of skewness in $[\mathrm{Fe} / \mathrm{H}]$ are positive. For stellar populations of $\tau>8 \mathrm{Gyr}$ at $|Z|<1.5 \mathrm{kpc}$, both the radial gradients of skewness and of mean values of $[\mathrm{Fe} / \mathrm{H}]$ are nearly flat. This suggests that the radial migration is clearly in operation and has affected the profiles of the $[\mathrm{Fe} / \mathrm{H}]$ distributions of stellar populations with significant radial $[\mathrm{Fe} / \mathrm{H}]$ gradients. The absence of the radial variations of the profiles of the $[\mathrm{Fe} / \mathrm{H}]$ distributions for stellar populations of $\tau>8 \mathrm{Gyr}$ is likely caused by the absence of any significant radial $[\mathrm{Fe} / \mathrm{H}]$ gradients of those stellar populations. Fig. 17 also shows that the radial $[\alpha / \mathrm{Fe}]$ gradients of stellar populations of $\tau>8 \mathrm{Gyr}$ are negative, while the radial skewness gradients in $[\alpha / \mathrm{Fe}]$ are positive. The radial variations of profiles of $[\alpha / \mathrm{Fe}]$ for stellar populations of $\tau>8 \mathrm{Gyr}$ are likely the combined conse- 


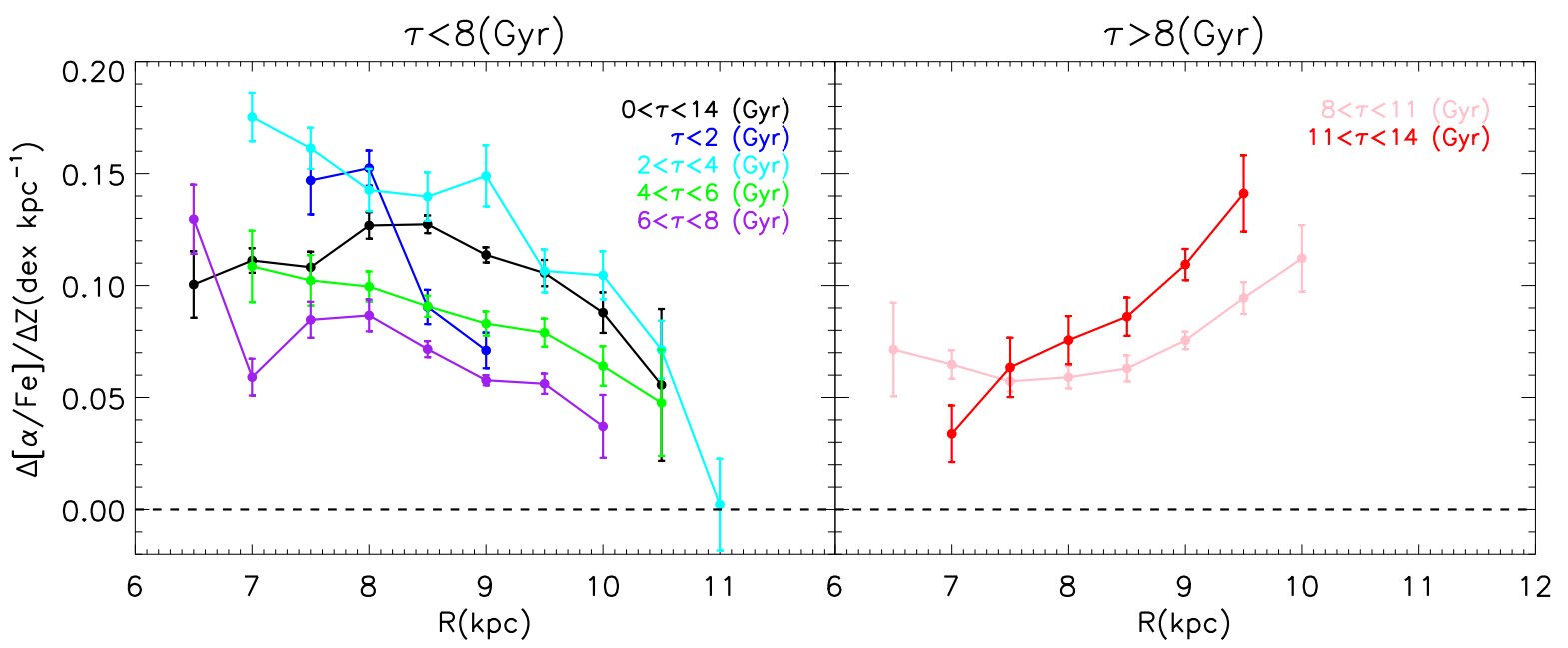

Figure 12. Vertical $[\alpha / \mathrm{Fe}]$ gradients as a function of $R$ derived from mono-age stellar populations, presented with different colours, as marked in the plot. The horizontal dashed lines delineate a constant vertical $[\alpha / \mathrm{Fe}]$ gradient value of 0.0 dex kpc ${ }^{-1}$. Vertical [ $\left.\alpha / \mathrm{Fe}\right]$ gradients flatten as $R$ increases for populations of $\tau<8 \mathrm{Gyr}$ (left panel), but steepen as $R$ increases for populations of $\tau>8 \mathrm{Gyr}$ (right panel)

quence of the presence of negative radial $[\alpha / \mathrm{Fe}]$ gradients and the radial migration processes.

In short, negative radial $[\mathrm{Fe} / \mathrm{H}]$ or $[\alpha / \mathrm{Fe}]$ gradients, combined with the radial migration processes, which change the motion radii of the stars, are responsible for the observed radial variations of the profiles of the $[\mathrm{Fe} / \mathrm{H}]$ and $[\alpha / \mathrm{Fe}]$ distributions for young and old populations, respectively.

Generally, there are two types of radial migrations, the blurring and churning (e.g. Schönrich \& Binney 2009). In the churning process, a star changes its orbit radii due to a gain/loss of angular momentum. In the blurring process, the angular momentum of a star is conserved and its epicycle amplitude changes. In the remaining parts of this Section, we will further discuss the effects of the blurring process on the metallicity distributions of the mono-age stellar populations. First, we integrate the orbit of each star, assuming a Galactic gravitational potential given by Gardner \& Flynn (2010), for a long enough time and derive the average $R$ and $|Z|$, i.e., $<R>$ and $<|Z|>$. We then examine the distributions as a function of the guiding centre radius $\left(R_{\mathrm{g}}=\langle R>)\right.$ and $|Z|$, rather than $R$ and $|Z|$. The results seem to rule out any significant effects of blurring in play.

Fig 18 shows the radial and vertical $[\mathrm{Fe} / \mathrm{H}]$ and $[\alpha / \mathrm{Fe}]$ gradients estimated in the $R-|Z|$ and $R_{\mathrm{g}}-|Z|$ planes for the different mono-age stellar populations. For stellar populations of $\tau>8 \mathrm{Gyr}$, the radial $[\mathrm{Fe} / \mathrm{H}]$ and $[\alpha / \mathrm{Fe}]$ gradients estimated in the $R-|Z|$ plane are flatter and steeper than those derived in the $R_{\mathrm{g}}-|Z|$ plane, respectively. In the regions of low $|Z|$, the radial $[\mathrm{Fe} / \mathrm{H}]$ gradients derived in $R$ and $|Z|$ plane are steeper than those deduced in the $R_{\mathrm{g}}-|Z|$ plane for the stellar populations of $\tau<8 \mathrm{Gyr}$. The radial $[\alpha / \mathrm{Fe}]$ gradients for the stellar populations of $4<\tau<8 \mathrm{Gyr}$ derived in the $R-|Z|$ plane are steeper than those derived in the $R_{\mathrm{g}}-|Z|$ plane. The results suggest that the blurring process may steepen the radial $[\mathrm{Fe} / \mathrm{H}]$ gradients for populations of $\tau<8 \mathrm{Gyr}$, and the radial $[\alpha / \mathrm{Fe}]$ gradients for all stellar populations, while its effects on the radial $[\mathrm{Fe} / \mathrm{H}]$ gradients are likely to be small or negligible for populations of $\tau>8 \mathrm{Gyr}$. The results are opposite to the predictions of chemical evolution models (e.g. Schönrich \& Binney 2009) that suggest that the radial migration will lead to the flattening of the radial metallicity gradients. While the chemical evolution models always assume a smooth radial stellar number (mass) distribution, the real disc shows complicated structure, and this may partly account for the difference. It is noted that Schönrich \& Binney (2009) considered both two types of radial migration processes, which may also produce the difference. The vertical $[\mathrm{Fe} / \mathrm{H}]$ and $[\alpha / \mathrm{Fe}]$ gradients derived in the $R-|Z|$ plane and those derived in the $R_{\mathrm{g}}-|Z|$ plane are quite identical to each other with an exception that the vertical $[\mathrm{Fe} / \mathrm{H}]$ gradient at $R-|Z|$ plane is flatter than that at $R_{\mathrm{g}}-|Z|$ plane for population of $\tau<4 \mathrm{Gyr}$.

As discussed in Section 4 , the skewness of the $[\mathrm{Fe} / \mathrm{H}]$ and [ $\alpha / \mathrm{Fe}]$ distributions is a function of $R$ for the young and old populations, respectively. In order to check whether the blurring is the main process producing the radial trends of $[\mathrm{Fe} / \mathrm{H}]$ and $[\alpha / \mathrm{Fe}]$ distributions, we plot the profiles of the $[\mathrm{Fe} / \mathrm{H}]$ and $[\alpha / \mathrm{Fe}]$ distributions at different $R$ and $R_{\mathrm{g}}$ bins for the young and old populations at relatively low $|Z|$ regions, respectively, in Fig 19 In Fig 19 , we plot the distributions of $[\mathrm{Fe} / \mathrm{H}]$ of stellar populations of $4<\tau<6 \mathrm{Gyr}$ and $11<\tau<14 \mathrm{Gyr}$ in order to check whether the blurring effect affects the $[\mathrm{Fe} / \mathrm{H}]$ distributions of different mono-age populations differently. For the distributions of $[\alpha / \mathrm{Fe}]$, we only show the results of stellar populations of $8<\tau<11 \mathrm{Gyr}$ at $0.3<|Z|<1.5 \mathrm{kpc}$ in which there are enough number of stars. Fig 19 shows that the profiles of the $[\mathrm{Fe} / \mathrm{H}]$ and $[\alpha / \mathrm{Fe}]$ distributions also vary with $R_{\mathrm{g}}$ for the young and old populations, respectively. This suggests that the radial variations of the skewness is not a consequence of the blurring process. Hayden et al. (2015) argue that a chemical evolution model considering the churning rather than the blurring process can reproduce the radial variations of the observed profiles of the $[\mathrm{Fe} / \mathrm{H}]$ distributions. Alternatively, the effects of blurring must be much smaller than those of churnning. Their conclusions are consistent with our results.

Fig 19 also shows that the dispersions of the metallicity distributions derived in the $R-|Z|$ plane are larger than those derived in the $R_{\mathrm{g}}-|Z|$ plane. This suggests that the blurring process may have increased the dispersions of the metallicity distributions for both the young and old stellar populations. Comparing the top and bottom panels in the third column of the Figure, one finds that there are more high- $\alpha$ stars in the $7<R<8 \mathrm{kpc}$ and $0.3<|Z|<1.5 \mathrm{kpc}$ bin than in the $7<R_{\mathrm{g}}<8 \mathrm{kpc}$ and $0.3<|Z|<1.5 \mathrm{kpc}$ bin. By 

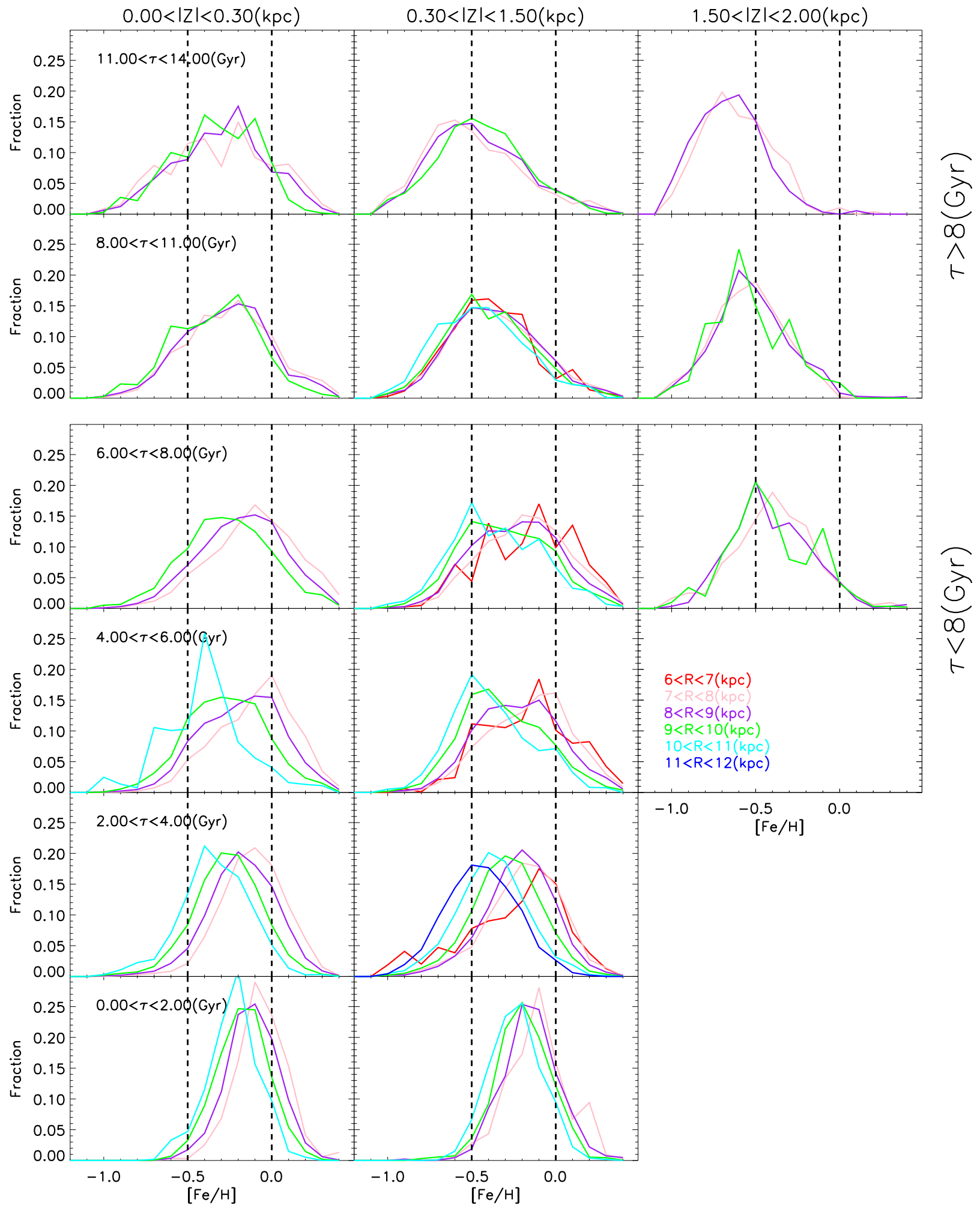

Figure 13. Distributions of $[\mathrm{Fe} / \mathrm{H}]$ in different $R,|Z|$ and $\tau$ bins. The $X$ axis show the values of $[\mathrm{Fe} / \mathrm{H}], Y$ show the relative stellar numbers in different $[\mathrm{Fe} / \mathrm{H}]$ bins. The left, middle and right panels show the results derived from stars at $|Z|<0.3 \mathrm{kpc}, 0.3<|Z|<1.5 \mathrm{kpc}, 1.5<|Z|<2.0 \mathrm{kpc}$, respectively. The age ranges of the mono-age stellar populations are marked in each panel. Results for different radial bins are presented by lines of different colours, as labeled in the lower right panel of the plot. The vertical dashed lines delineate constant $[\mathrm{Fe} / \mathrm{H}]$ values of -0.5 and 0.0 dex. The profiles of $[\mathrm{Fe} / \mathrm{H}]$ distributions show significantly radial variations for young stellar populations $(\tau<8 \mathrm{Gyr})$, but no clear radial variations for old stellar populations $(\tau>8 \mathrm{Gyr})$. 


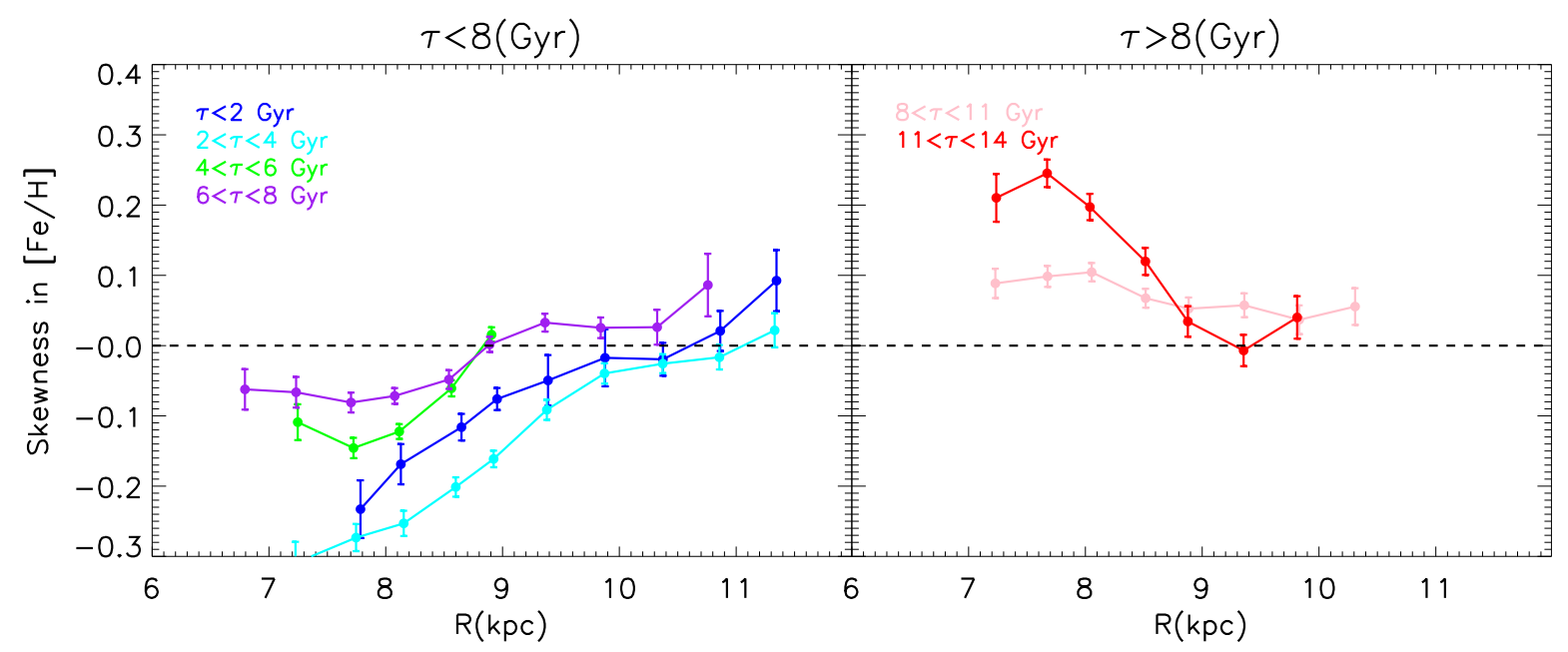

Figure 14. Skewness as a function of $R$ for the mono-age stellar populations (at $|Z|<1.5 \mathrm{kpc}$ ), presented with different colours, as marked in the plot. The skewness of stellar populations of $\tau<8 \mathrm{Gyr}$ (left panel) increases as $R$ increases, while that of stellar populations of $\tau>8 \mathrm{Gyr}$ (right panel) decreases or is nearly invariant as $R$ increases. The results for stellar population of $\tau>11 \mathrm{Gyr}$ should be taken with caution due to the possible young dwarf star contamination.

examining the $R_{\mathrm{g}}$ distribution of stars in $7<R<8 \mathrm{kpc}$, we find that there are much more stars of $R_{\mathrm{g}}<7 \mathrm{kpc}$ than stars of $R_{\mathrm{g}}>8$ kpc. Clearly, those high- $\alpha$ stars originate from the inner disc and move to their current positions through the blurring process.

\section{CONSTRAINING THE FORMATION AND EVOLUTION OF THE DISC(S)}

\subsection{The formation scenario of the disc at an early epoch}

The radial and vertical $[\mathrm{Fe} / \mathrm{H}]$ and $[\alpha / \mathrm{Fe}]$ gradients of the oldest stellar populations provide strong constrains on the formation scenario of the disc at early epoch. The oldest stellar populations $(\tau>$ $11 \mathrm{Gyr})$ show nearly flat or marginally positive radial $[\mathrm{Fe} / \mathrm{H}]$ gradients. It suggests that the oldest stellar populations formed from gas that was originally well-mixed in the radial direction in a violent, fast formation process. A fast, highly-turbulent gas-rich merger formation scenario as proposed by Brook et al. (2004 2005) suggests that the thick disc formed so fast (with a timescale shorter than the life time of Type Ia supernovae ( $\mathrm{SNe}$ Ia), which produce most of the iron elements) at an early epoch that there should be no $[\mathrm{Fe} / \mathrm{H}]$ gradients in the radial direction, consistent with our results of the oldest stellar populations.

On the other hand, we find that there are significant negative vertical $[\mathrm{Fe} / \mathrm{H}]$ gradients and negative radial $[\alpha / \mathrm{Fe}]$ gradients for the oldest stellar populations. This suggests that the formation of the oldest disc (meaning here the disc older than $11 \mathrm{Gyr}$ ) can not be too fast as it needs enough time to develop vertical $[\mathrm{Fe} / \mathrm{H}]$ gradients and negative radial $[\alpha / \mathrm{Fe}]$ gradients. The timescale of disc formation should be longer than the life time of Type II supernovae (SNe II). SNe II produce most of the $\alpha$ elements. Before $11 \mathrm{Gyr}$, several generations of stars have evolved and changed the $[\alpha / \mathrm{Fe}]$ of the disc. Higher star formation rates (SFRs) (discussed in the Section 6.2) in the inner disc produce more exploring of SNe II in the inner disc and the negative radial $[\alpha / \mathrm{Fe}]$ gradient presented in the current work.

In conclusion, the disc formed from an originally well-mixed gas in a violent, fast formation process at early epoch. The timescale of the process is shorter than the life time of SNe Ia, but longer than the life time of SNe II. In the evolution of the oldest disc, the vertical heating play an important role.

\subsection{The "inside-out" and "upside-down" disc formation scenario}

Our data shows negative radial $[\mathrm{Fe} / \mathrm{H}]$ gradients for the stellar populations younger than $11 \mathrm{Gyr}$ and negative radial $[\alpha / \mathrm{Fe}]$ gradients for the oldest stellar populations, which is a consequence of the "inside-out" disc formation scenario (Matteucci \& Francois 1989, Schönrich \& McMillan 2017). In this scenario, SFRs in the inner disc are higher than in the outer disc, yielding more metals in the inner disc than in the outer disc and the negative radial $[\mathrm{Fe} / \mathrm{H}]$ gradients. As discussed in the Section 6.1, the "inside-out" disc formation scenario is also responsible for the negative radial $[\alpha / \mathrm{Fe}]$ gradients for the oldest stellar populations. At early epoch, as $R$ decreases, the SFRs increase, yielding more exploding of SNe II, more $\alpha$-elements and higher $[\alpha / \mathrm{Fe}]$ in the inner disc.

All the mono-age stellar populations show negative vertical $[\mathrm{Fe} / \mathrm{H}]$ gradients. This is consistent with the theoretical predictions of Kawata et al. (2017), who consider among others also monoage stellar populations formed in a flaring, star-forming disc. In this scenario, stars in the outer regions can reach to higher vertical heights than those stars in the inner regions because of their higher vertical energies, which producing a flaring disc. Radial mixing then yield negative vertical $[\mathrm{Fe} / \mathrm{H}]$ gradients (Kawata et al. 2017, Ciucă et al. 2018). If the radial migration process is slow, old stars have more time to migrate to outer disc, producing a steeper (negative) vertical $[\mathrm{Fe} / \mathrm{H}]$ gradients for the old stellar populations. Again this is consistent with our finding. On the other hand, if this scenario does work, then one would expect negative vertical $[\alpha / \mathrm{Fe}]$ gradients, not consistent with our finding that all the monoage stellar populations exhibit positive vertical $[\alpha / \mathrm{Fe}]$ gradients. Our results thus prefers the "upside-down" disc formation scenario. This scenario suggests that the height of the star-forming gas layer contracts as the stellar mass of the disc grows (Bird et al. 2013 . Bournaud, Elmegreen \& Martig 2009). And as the metal-rich and 

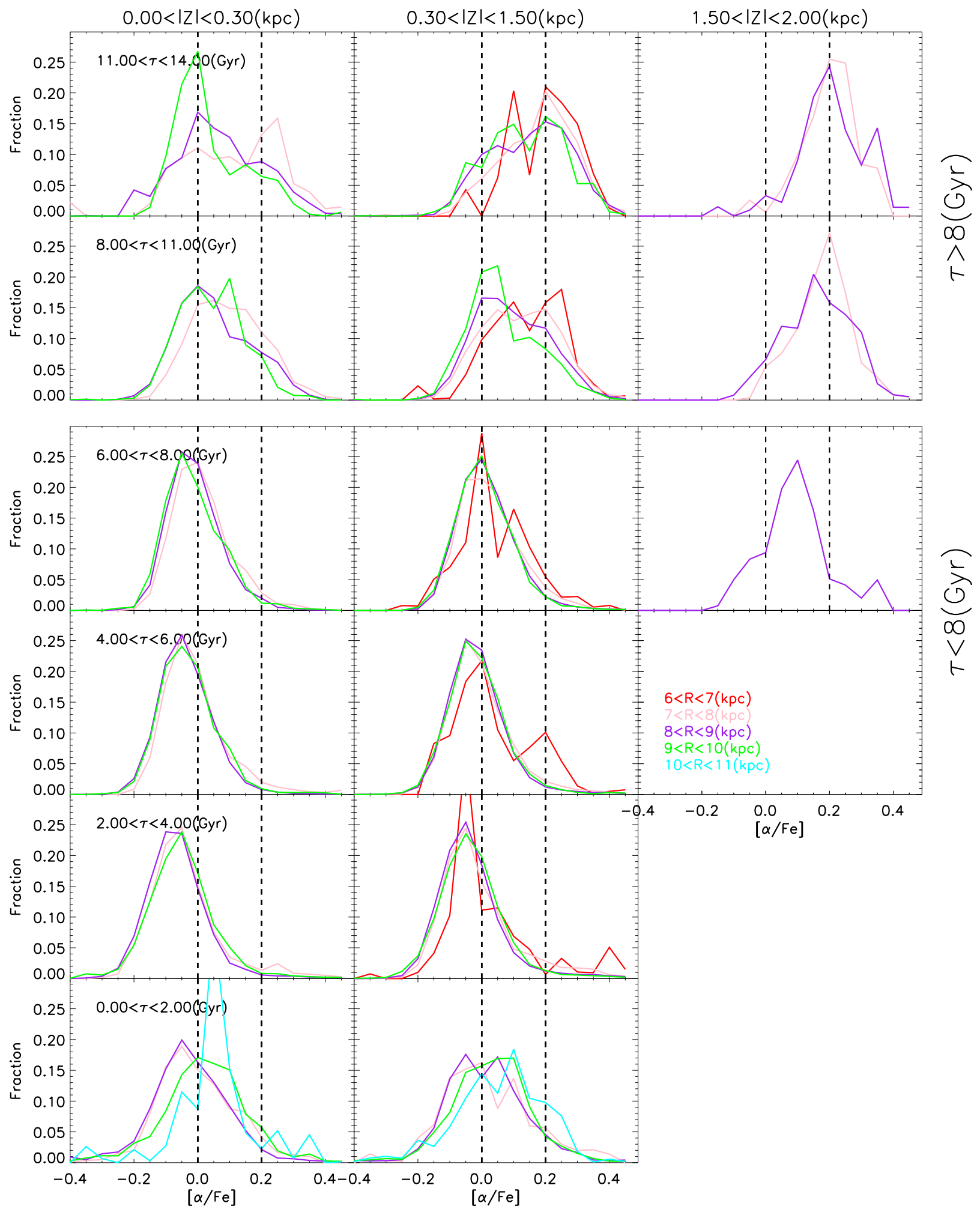

Figure 15. Same as Fig 13 but for $[\alpha / \mathrm{Fe}]$. The vertical dashed lines delineate constant $[\alpha / \mathrm{Fe}]$ values of 0.0 and 0.2 dex. The results for stellar population of $\tau<2 \mathrm{Gyr}$ should be taken with caution as about $10-20$ per cent $[\alpha / \mathrm{Fe}]$ measurements of those stars may be incorrect. 


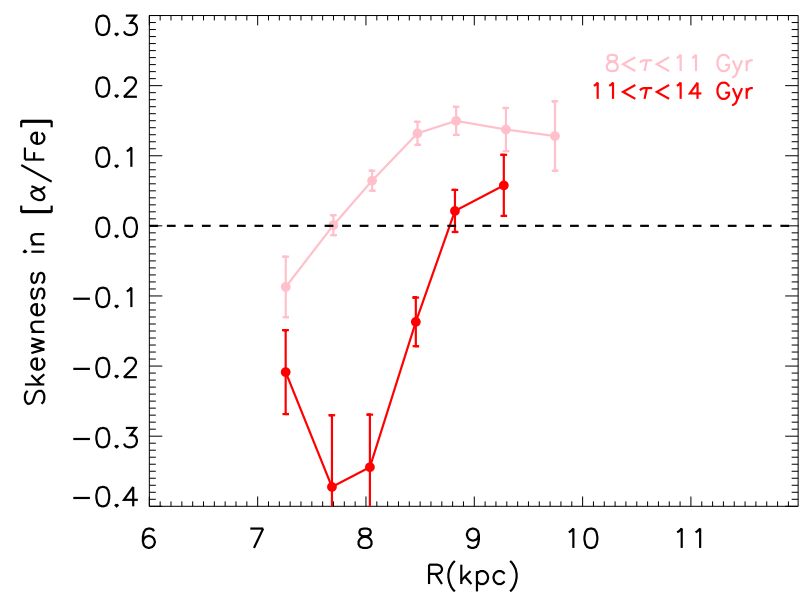

Figure 16. Same as Fig 14 but for $[\alpha / \mathrm{Fe}]$. The skewness of $[\alpha / \mathrm{Fe}]$ distributions of old stellar populations ( $\tau>8 \mathrm{Gyr}$ ) increase as $R$ increases.

$[\alpha / \mathrm{Fe}]$-poor gas settles down to the Galactic plane, it produces negative vertical $[\mathrm{Fe} / \mathrm{H}]$ gradients and positive vertical $[\alpha / \mathrm{Fe}]$ gradients.

\subsection{The superposition of two discs}

For the low- $|Z|$ regions, our current study finds that the radial $[\alpha / \mathrm{Fe}]$ gradients steepen with increasing age, while for the high- $|Z|$ regions, the gradients flatten with increasing age. The results are the consequence of superposition of two discs, a low- $\alpha$, "thin" disc of a large scale length, and a high- $\alpha$, "thick" disc of a small scale length. The relative fraction of the "thin" and the "thick" disc are different for different mono-age stellar populations, younger stellar populations contain more low- $\alpha$, "thin" disc stars. In the low- $|Z|$ regions, low- $\alpha$ "thin" disc stars dominate both the inner and outer discs (see Fig 11 for young stellar populations, leading to relatively flat gradients. Populations of older ages contain more $\alpha$-rich "thick" disc stars in the inner disc than in the outer disc (see Fig[11, yielding relatively steeper (negative) gradients. In the high- $|Z|$ regions, "thick" disc, $\alpha$-rich stars dominate in the inner disc (see Fig 11) for all the mono-age stellar populations. It seems that the $\alpha$-rich disc contains stars as young as $2-4 \mathrm{Gyr}$. Those young $\alpha$-rich stars are either a consequence of a sustained star formation process of the thick disc, or intrinsically old stars whose ages have been incorrectly underestimated-some of them may well be blue straggles (Xiang et al. 2017a). In the outer disc, young stars are dominated by "thin" disc, $\alpha$-poor stars (see Fig 11 . This produces relatively steeper negative radial $[\alpha / \mathrm{Fe}]$ gradients for young stars. Likewise, old stars in the outer disc are dominated by "thick" disc, $\alpha$-rich stars, leading to relatively flat negative radial $[\alpha / \mathrm{Fe}]$ gradients for old stars. The superposition of two discs can also naturally explain the finding that the positive vertical $[\alpha / \mathrm{Fe}]$ gradients flatten with increasing $R$ for the stellar populations of $\tau<8 \mathrm{Gyr}$, while steepen with increasing $R$ for the stellar populations of $\tau>8 \mathrm{Gyr}$.

\section{SUMMARY}

In this work, two samples, a sample consisting of 453,188 LAMOST MSTO-SG stars of spectral SNRs > 20 (MSTO-SG-SNR20) and another consisting of 291,126 similar stars of spectral SNRs > 50 (MSTO-SG-SNR50), are built to study the $[\mathrm{Fe} / \mathrm{H}]$ and $[\alpha / \mathrm{Fe}]$ distributions of mono-age stellar populations, respectively. Here, we have carried out for the first time a systematic study of the profiles of $[\mathrm{Fe} / \mathrm{H}]$ distributions, the radial and vertical $[\alpha / \mathrm{Fe}]$ gradients and the profiles of $[\alpha / \mathrm{Fe}]$ distributions of mono-age stellar populations. For the MSTO-SG-SNR20 sample, estimates of effective temperature, surface gravity, metallicity, radial velocity and absolute magnitudes are estimated to be accurate to $150 \mathrm{~K}, 0.2 \mathrm{dex}, 0.15$ dex, $5 \mathrm{~km} \mathrm{~s}^{-1}$ and $0.5-0.6 \mathrm{mag}$, respectively. Estimates of $[\alpha / \mathrm{Fe}]$ are estimated to be accurate to 0.05 dex for MSTO-SG-SNR50 sample stars. For the two MSTO-SG samples, age and distance errors are about 30 and 17 percent, respectively. The main results presented in this work are the following:

(a) The main results on the radial and vertical $[\mathrm{Fe} / \mathrm{H}]$ and $[\alpha / \mathrm{Fe}]$ gradients:

- Radial $[\mathrm{Fe} / \mathrm{H}]$ gradients derived from stars of $\tau<11 \mathrm{Gyr}$ are negative, and vary with $|Z|$ and $\tau$. The gradients flatten with increasing $|Z|$. Radial $[\mathrm{Fe} / \mathrm{H}]$ gradients derived from stars of $\tau>11 \mathrm{Gyr}$ are $\sim-0.00 \pm 0.03 \mathrm{dex} \mathrm{kpc}^{-1}$, and vary little with $|Z|$. After being nearly flat at the earliest epoch of the disc formation, the radial $[\mathrm{Fe} / \mathrm{H}]$ gradients steepen with decreasing age, reaching maxima between $4<\tau<6 \mathrm{Gyr}$, and then flatten again.

- There are negative vertical $[\mathrm{Fe} / \mathrm{H}]$ gradients, that vary with $R$ and $\tau$. The gradients flatten with increasing $R$, and steepen with increasing age at $R>8 \mathrm{kpc}$.

- Radial $[\alpha / \mathrm{Fe}]$ gradients derived for the stellar populations of $\tau<4$ Gyr decrease with increasing $|Z|$. The gradients are nearly flat for the stellar populations of $4<\tau<6$ Gyr and invariant with $|Z|$. There are negative gradients for the stellar populations of $\tau>6$ Gyr. The gradients is a function of age. They steepen with increasing age in the low- $|Z|$ regions and flatten with increasing age in the high- $|Z|$ regions.

- There are positive vertical $[\alpha / \mathrm{Fe}]$ gradients that vary with $R$ and $\tau$. The gradients flatten with increasing $R$ for the stellar populations of $\tau<8 \mathrm{Gyr}$ and steepen with increasing $R$ for the stellar populations of $\tau>8 \mathrm{Gyr}$.

(b)The main results of profiles on the $[\mathrm{Fe} / \mathrm{H}]$ and $[\alpha / \mathrm{Fe}]$ distributions:

- The profiles of the $[\mathrm{Fe} / \mathrm{H}]$ distributions vary with $R$ for the young stellar populations of $\tau<8$ Gyr close to the Galactic plane $(|Z|<1.5 \mathrm{kpc})$. They show negatively skewed distributions in the inner disc and positively skewed distributions in the outer disc. The profiles of the distributions of the young stellar populations in the high- $|Z|(|Z|>1.5 \mathrm{kpc})$ regions show only marginal variations with $R$. The profiles are nearly the same in all $R$ bins for the stellar populations of $\tau>8 \mathrm{Gyr}$ in all $|Z|$ regions.

- The profiles of the $[\alpha / \mathrm{Fe}]$ distributions change with $R$ for the old stellar populations of $\tau>8 \mathrm{Gyr}$ in all $|Z|$ regions. Old stellar populations have negatively skewed distributions in the inner disc and positively skewed distributions in the outer disc. The profiles show weak radial variations for populations of $\tau<8 \mathrm{Gyr}$.

Radial and vertical $[\mathrm{Fe} / \mathrm{H}]$ gradients and their variations with radius and height deduced here for mono-age populations are quite similar with those obtained by Xiang et al. (2015b). For the stellar populations of $\tau<8 \mathrm{Gyr}$, the observed radial variations of the profiles of the $[\mathrm{Fe} / \mathrm{H}]$ distributions also match very well with those derived from the $\alpha$-poor stellar populations by Hayden et al. (2015), who suggest that radial migration (especially the churning process) is an important process in the Galactic disc formation and evolu- 


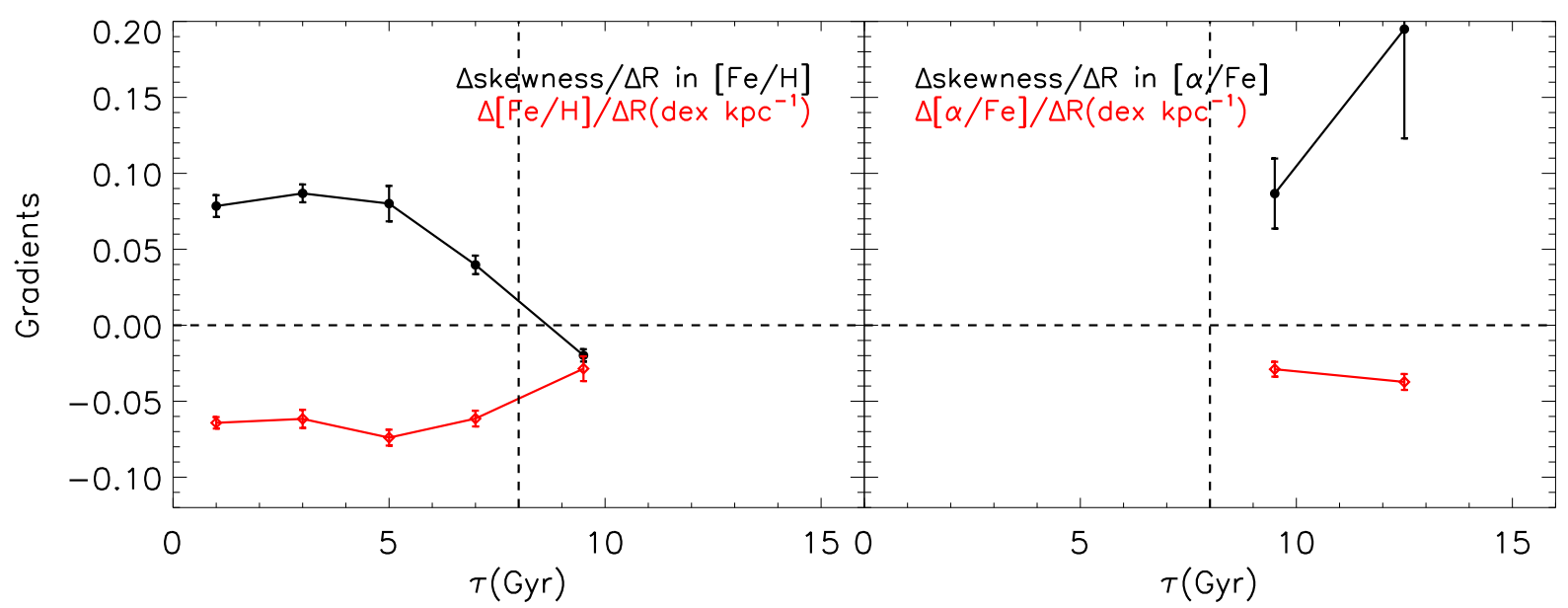

Figure 17. The radial gradients of skewness (black dots and lines) and mean values (red dots and lines) of [Fe/H] (left panel) and [ $\alpha / \mathrm{Fe}]($ right panel) as a function of age for stellar populations at $|Z|<1.5 \mathrm{kpc}$. The horizontal lines delineate a constant gradient value of 0.0 . The vertical lines delineate a constant age of $8 \mathrm{Gyr}$. The variations of the radial skewness gradients with age are opposite to the variations of the radial gradients of the mean values.
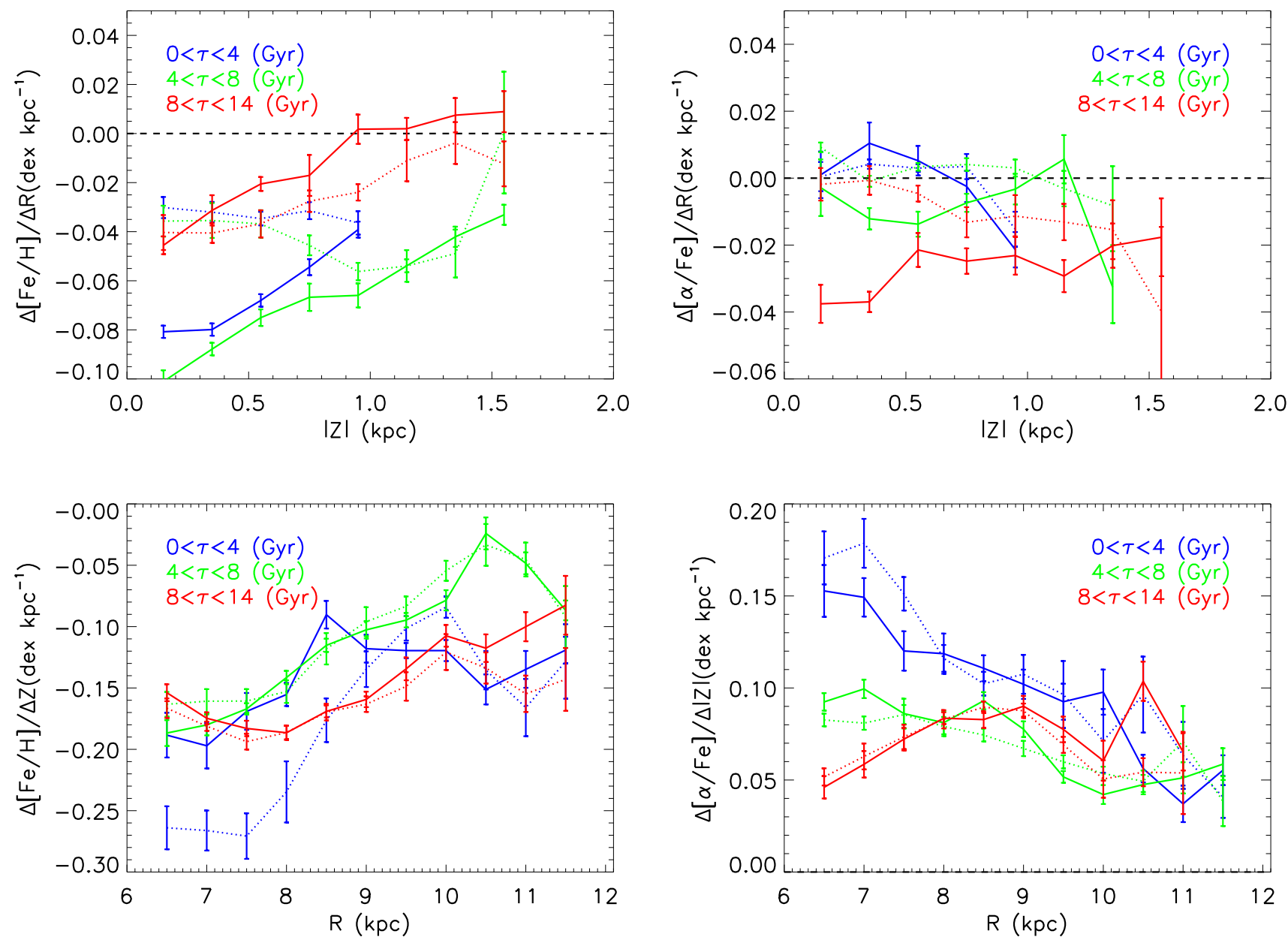

Figure 18. Radial (top panels) and vertical (bottom panels) $[\mathrm{Fe} / \mathrm{H}]$ (left panels) and $[\alpha / \mathrm{Fe}]$ (right panels) gradients. Solid lines show gradients estimated in the $R-|Z|$ plane, while dotted lines show those estimated in the $R_{\mathrm{g}}-|Z|$ plane. Different colors show the results of the different mono-age stellar populations, as marked in the panels. The horizontal lines delineate a constant gradient value of $0.0 \mathrm{dex} \mathrm{kpc}^{-1}$. 

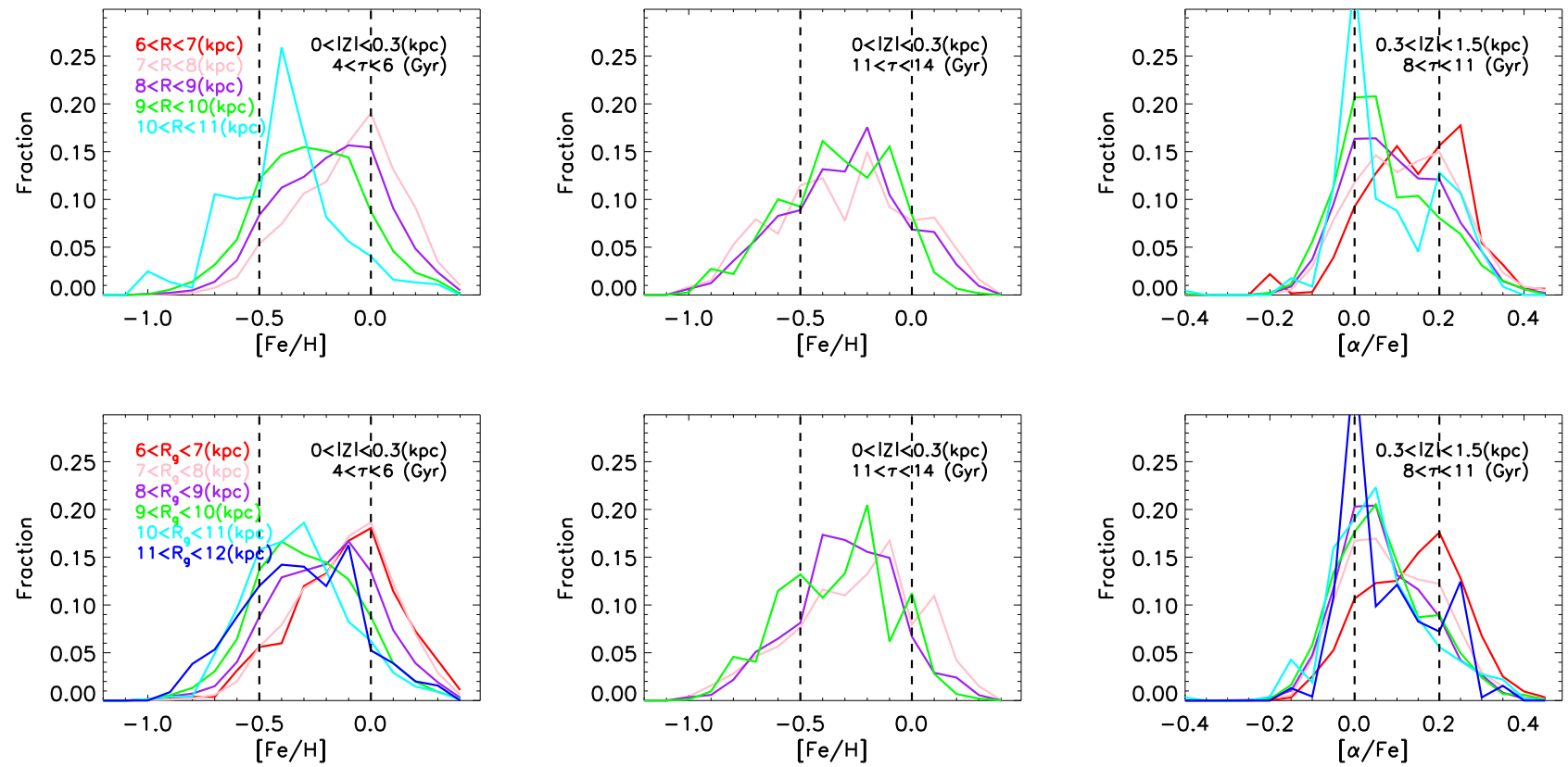

Figure 19. $[\mathrm{Fe} / \mathrm{H}]$ (first and second columns) and $[\alpha / \mathrm{Fe}]$ (third column) distributions. The top panels show the distributions derived in the $R-|Z|$ plane, while those in the bottom show the results derived in the $R_{\mathrm{g}}-|Z|$ plane. Panels in the first column show the $[\mathrm{Fe} / \mathrm{H}]$ distributions derived for the stellar populations of $4<\tau<6$ Gyr close to the Galactic plane. The panels in the second column show the $[\mathrm{Fe} / \mathrm{H}]$ distributions derived for the stellar populations of $11<\tau<14$ Gyr close to the Galactic plane. The panels in the third column show the $[\alpha / \mathrm{Fe}]$ distributions for the stellar populations of $8<\tau<11 \mathrm{Gyr}$ of height $0.3<|Z|<1.5 \mathrm{kpc}$. The vertical lines delineate constant $[\mathrm{Fe} / \mathrm{H}]$ values of -0.5 and $0.0 \mathrm{dex}$, and constant $[\alpha / \mathrm{Fe}]$ values of 0.0 and $0.2 \mathrm{dex}$.

tion. The radial variations of the profiles of the $[\mathrm{Fe} / \mathrm{H}]$ distributions derived here for the stellar populations of $\tau>8 \mathrm{Gyr}$ also match very well with those derived from the $\alpha$-rich stellar populations by Hayden et al. (2015).

By examining the metallicity distributions in the $R,|Z|$ and $\tau$ space, as well as in the $R_{\mathrm{g}},|Z|$ and $\tau$ space, we find that the churning process rather than the blurring process is the main reason responsible for the observed radial variations of the profiles of the metallicity distributions, consistent with the results of Hayden et al. (2015). The blurring process may have increased the widths of the profiles of the metallicity distributions.

Benefiting from the wide age and large volume coverage of our MSTO-SG samples, we have mapped the metallicity distributions in different $R,|Z|$ and $\tau$ bins. The results provide strong constraints of formation and evolution of the Galactic disc(s). Our measured radial $[\mathrm{Fe} / \mathrm{H}]$ and $[\alpha / \mathrm{Fe}]$ gradients support the "inside-out" disc formation scenario, while the vertical $[\mathrm{Fe} / \mathrm{H}]$ and $[\alpha / \mathrm{Fe}]$ gradients are consistent with the "upside-down" disc formation scenario. Variations with age of the radial $[\alpha / \mathrm{Fe}]$ gradients suggest that the Galactic disc is a superposition of two discs, a low- $\alpha$, "thin" disc of a large scale length, and a high- $\alpha$, "thick" disc of a small scale length. The measured values of $[\alpha / \mathrm{Fe}]$ provide strong constraints of the SNe II/Ia ratio. The clearly observed negative radial $[\alpha / \mathrm{Fe}]$ gradients derived from the populations of $\tau>8 \mathrm{Gyr}$ suggest that $\mathrm{SNe}$ II/Ia ratio is larger in the outer disc than that in the inner disc at early epoch of the disc formation.

\section{ACKNOWLEDGEMENTS}

We appreciate the helpful comments of the anonymous referee. This work is supported by National Key Basic Research Program of China 2014CB845700, the NSFC grant 11703035 and the Joint Funds of the National Natural Science Foundation of China (Grant No. U1531244 and U1331120. Guoshoujing Telescope (the Large Sky Area Multi-Object Fiber Spectroscopic Telescope LAMOST) is a National Major Scientific Project built by the Chinese Academy of Sciences. Funding for the project has been provided by the National Development and Reform Commission. LAMOST is operated and managed by the National Astronomical Observatories, Chinese Academy of Sciences. This work is also supported by National Natural Science Foundation of China (Grant No. 11473001).

\section{REFERENCES}

Anders F. et al., 2014, A\&A, 564, A115

Andrews B. H., Weinberg D. H., Schönrich R., Johnson J. A., 2017, ApJ, 835, 224

Andrievsky S. M. et al., 2002, A\&A, 381, 32

Balser D. S., Rood R. T., Bania T. M., Anderson L. D., 2011, ApJ, 738, 27

Bird J. C., Kazantzidis S., Weinberg D. H., Guedes J., Callegari

S., Mayer L., Madau P., 2013, ApJ, 773, 43

Boeche C. et al., 2014, A\&A, 568, A71

Boeche C. et al., 2013, A\&A, 559, A59

Boeche C., Smith M. C., Grebel E. K., Zhong J., Hou J. L., Chen L., Stello D., 2018, AJ, 155, 181

Bournaud F., Elmegreen B. G., Martig M., 2009, ApJ, 707, L1

Brook C. B., Gibson B. K., Martel H., Kawata D., 2005, ApJ, 630, 298

Brook C. B., Kawata D., Gibson B. K., Freeman K. C., 2004, ApJ, 612,894 
Casagrande L., Schönrich R., Asplund M., Cassisi S., Ramírez I., Meléndez J., Bensby T., Feltzing S., 2011, A\&A, 530, A138

Chen B.-Q. et al., 2017, MNRAS, 464, 2545

Chen B.-Q., Liu X.-W., Yuan H.-B., Xiang M.-S., Huang Y., Wang

C., Zhang H.-W., Tian Z.-J., 2018, MNRAS, 476, 3278

Chen L., Hou J. L., Wang J. J., 2003, AJ, 125, 1397

Cheng J. Y. et al., 2012, ApJ, 746, 149

Ciucă I., Kawata D., Lin J., Casagrande L., Seabroke G., Cropper M., 2018, MNRAS, 475, 1203

Colavitti E., Matteucci F., Murante G., 2008, A\&A, 483, 401

Costa R. D. D., Uchida M. M. M., Maciel W. J., 2004, A\&A, 423, 199

Cui X.-Q. et al., 2012, Research in Astronomy and Astrophysics, 12,1197

Daflon S., Cunha K., 2004, ApJ, 617, 1115

Deng L.-C. et al., 2012, Research in Astronomy and Astrophysics, 12,735

Gardner E., Flynn C., 2010, MNRAS, 405, 545

Genovali K. et al., 2015, A\&A, 580, A17

Grisoni V., Spitoni E., Matteucci F., 2018, ArXiv e-prints

Hayden M. R. et al., 2015, ApJ, 808, 132

Hayden M. R. et al., 2014, AJ, 147, 116

Haywood M., Lehnert M. D., Di Matteo P., Snaith O., Schultheis M., Katz D., Gómez A., 2016, A\&A, 589, A66

Helmi A., Babusiaux C., Koppelman H. H., Massari D., Veljanoski J., Brown A. G. A., 2018, ArXiv e-prints

Henry R. B. C., Kwitter K. B., Jaskot A. E., Balick B., Morrison M. A., Milingo J. B., 2010, ApJ, 724, 748

Ho A. Y. Q. et al., 2017, ApJ, 836, 5

Huang Y. et al., 2015, Research in Astronomy and Astrophysics, 15,1240

Katz D., Soubiran C., Cayrel R., Barbuy B., Friel E., Bienaymé O., Perrin M.-N., 2011, A\&A, 525, A90

Kawata D., Grand R. J. J., Gibson B. K., Casagrande L., Hunt J. A. S., Brook C. B., 2017, MNRAS, 464, 702

Kobayashi C., Nakasato N., 2011, ApJ, 729, 16

Larson R. B., 1976, MNRAS, 176, 31

Lee Y. S. et al., 2015, AJ, 150, 187

Liu X.-W. et al., 2014, in IAU Symposium, Vol. 298, IAU Symposium, Feltzing S., Zhao G., Walton N. A., Whitelock P., eds., pp. 310-321

Loebman S. R., Roškar R., Debattista V. P., Ivezić Ž., Quinn T. R., Wadsley J., 2011, ApJ, 737, 8

Luck R. E., Kovtyukh V. V., Andrievsky S. M., 2006, AJ, 132, 902

Luo A.-L. et al., 2015, ArXiv e-prints

Magrini L., Sestito P., Randich S., Galli D., 2009, A\&A, 494, 95

Matteucci F., Francois P., 1989, MNRAS, 239, 885

Minchev I., Chiappini C., Martig M., 2014, A\&A, 572, A92

Minchev I., Famaey B., Quillen A. C., Dehnen W., Martig M., Siebert A., 2012, A\&A, 548, A127

Nandakumar G., Schultheis M., Hayden M., Rojas-Arriagada A., Kordopatis G., Haywood M., 2017, A\&A, 606, A97

Nordström B. et al., 2004, A\&A, 418, 989

Pezzulli G., Fraternali F., 2016, MNRAS, 455, 2308

Quillen A. C., Garnett D. R., 2001, in Astronomical Society of the Pacific Conference Series, Vol. 230, Galaxy Disks and Disk Galaxies, Funes J. G., Corsini E. M., eds., pp. 87-88

Quinn P. J., Hernquist L., Fullagar D. P., 1993, ApJ, 403, 74

Ren J.-J. et al., 2016, Research in Astronomy and Astrophysics, 16,45

Rix H.-W., Bovy J., 2013, A\&ARv, 21, 61
Roškar R., Debattista V. P., Quinn T. R., Stinson G. S., Wadsley J., 2008, ApJ, 684, L79

Schlesinger K. J. et al., 2012, ApJ, 761, 160

Schönrich R., Binney J., 2009, MNRAS, 396, 203

Schönrich R., McMillan P. J., 2017, MNRAS, 467, 1154

Sellwood J. A., Binney J. J., 2002, MNRAS, 336, 785

Siebert A. et al., 2011, AJ, 141, 187

Toyouchi D., Chiba M., 2018, ApJ, 855, 104

van den Bergh S., 1962, AJ, 67, 486

Xiang M. et al., 2017a, ApJS, 232, 2

Xiang M. et al., 2018, ArXiv e-prints

Xiang M. S. et al., 2015a, MNRAS, 448, 822

Xiang M.-S. et al., 2015b, Research in Astronomy and Astrophysics, 15, 1209

Xiang M.-S. et al., 2017b, MNRAS, 467, 1890

Yuan H.-B. et al., 2015, MNRAS, 448, 855

Yuan H. B., Liu X. W., Xiang M. S., 2013, MNRAS, 430, 2188

Zhao G., Zhao Y.-H., Chu Y.-Q., Jing Y.-P., Deng L.-C., 2012, Research in Astronomy and Astrophysics, 12, 723 